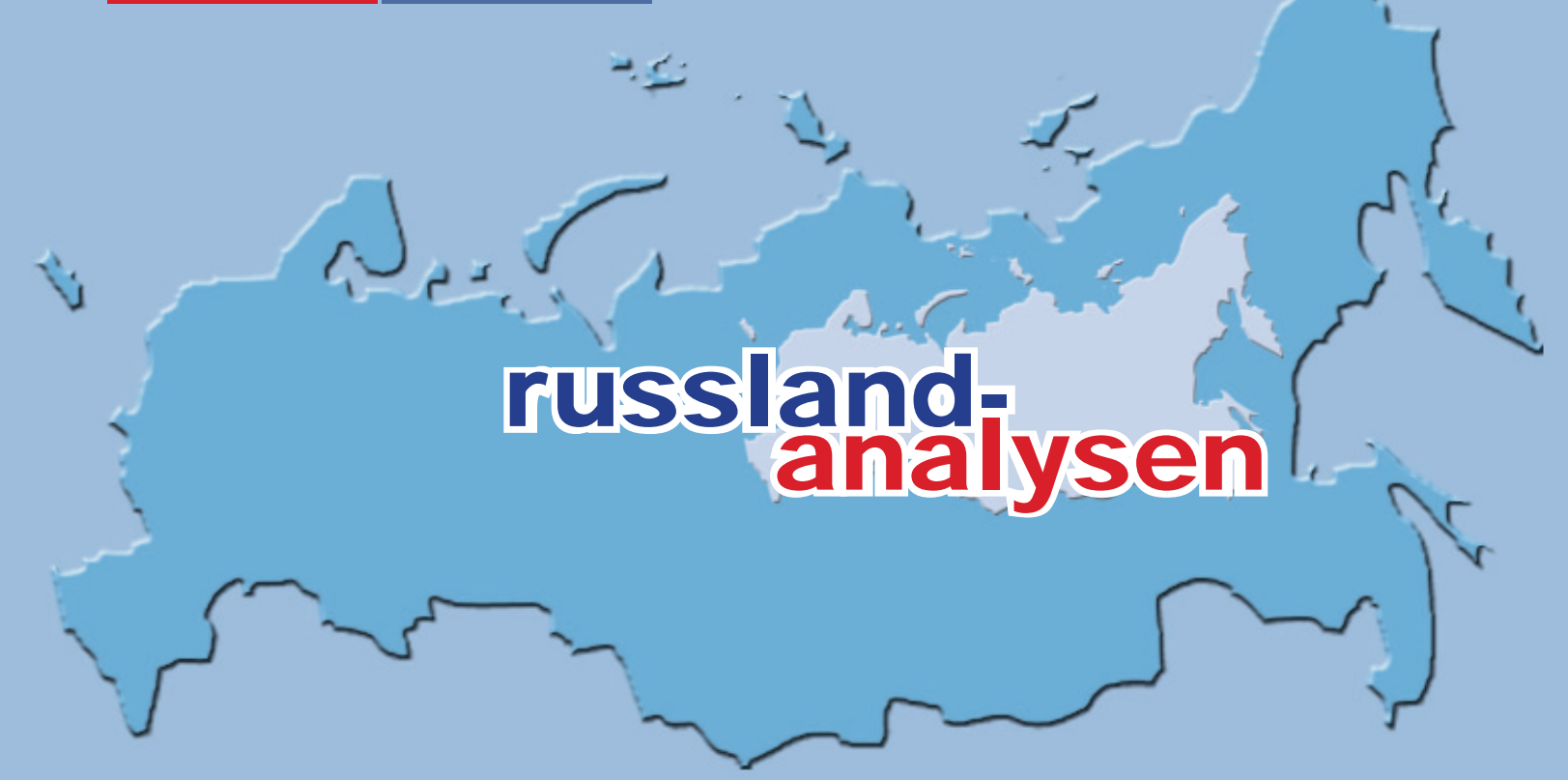

\title{
http://www.laender-analysen.de/russland/
}

\section{RUSSISCHE AFGHANISTANPOLITIK}

ANALYSE

Das Afghanistanproblem aus russischer Perspektive

ANALYSE

Die Bedeutung Afghanistans für Russland: Regionale oder globale Strategie?

Von Natasha Kuhrt, London

ANALYSE

Warum Russland in Afghanistan mit dem Westen kooperiert

Von David Krickus, Washington

DOKUMENTATION

UNO-Schätzungen über Produktion und Konsum von Opium und Heroin

CHRONIK

Der sowjetische Afghanistankrieg 1979-1989

DOKUMENTATION

Sowjetische Verluste in Afghanistan 1979-1989

UMFRAGE

Internationale Umfrage zum NATO-Einsatz in Afghanistan, Juli $2009 \quad 20$

Der sowjetische Afghanistankrieg in der russischen Erinnerung

CHRONIK

Vom 4. bis zum 17. Juni 2010

Die Russland-Analysen werden vom

Ost-Ausschuss der Deutschen Wirtschaft unterstützt.

\section{- Forschungsstelle Osteuropa an der Universitäł Bremen}




\section{Analyse}

\section{Das Afghanistanproblem aus russischer Perspektive}

Von Viktor Korgun, Moskau

\section{Zusammenfassung}

Erneut ist Afghanistan von zentraler außenpolitischer Bedeutung für Russland. Die instabile Lage im Lande, im Besonderen illegale Drogen, islamische Extremisten und die Korruption bedrohen die Stabilität Russlands und seiner strategischen Verbündeten in Zentralasien. Um sich mit diesen Herausforderungen zu befassen, sucht Russland eine aktivere Rolle beim Wiederaufbau und der Stabilisierung Afghanistans auf bilateraler, regionaler und internationaler Ebene zu spielen. Demnach verfolgen der Westen und Russland ein gemeinsames Interesse in Afghanistan und Moskau ist mehr als froh darüber, die westlich geführten internationalen Anstrengungen in Afghanistan kooperativ zu unterstützen.

\section{Die jüngste Geschichte der}

\section{russisch-afghanischen Beziehungen}

Über mehrere Jahrzehnte war Afghanistan ein zentrales Thema der sowjetischen Außenpolitik. Moskau stellte Afghanistan umfangreiche wirtschaftliche und ökonomische Unterstützung bereit und führte dann ab Ende der 1970er Jahre einen Krieg in Afghanistan, der letztendlich zum Niedergang der Sowjetunion 1991 beitrug. Nach der Auflösung der Sowjetunion sank in Moskau das Interesse an Afghanistan deutlich ab. In dieser Zeit kam es zu einem Bürgerkrieg, genauer zu einem Machtkampf zwischen den verschiedenen Gruppen der Mudschahidin, der zu einem Zerfall Afghanistans und dem Auftreten der politischen und militärischen Bewegung der Taliban führte. Nach der Absetzung des TalibanRegimes durch den NATO-Einsatz "Enduring Freedom" im Jahr 2001 entstand eine Situation, in der ein Wiederaufbau Afghanistans und der Übergang zu einer friedlichen und demokratischen Entwicklung möglich wurde. In der Folge ergab sich die Chance, die traditionell freundschaftlichen Beziehungen zwischen Russland und Afghanistan wieder herzustellen.

\section{Nach der Operation "Enduring Freedom"}

Die russische Führung unterstützte aktiv den Anti-Terror-Einsatz der NATO in Afghanistan. Sie entschied sich sogar dafür, sich der Stationierung von NATOTruppen in Militärbasen in Usbekistan, Tadschikistan und Kirgistan nicht entgegenzustellen. Im Rahmen der umfangreichen internationalen Unterstützung dieser Militäroperation stellte die russische Regierung humanitäre Hilfe für die afghanische Bevölkerung bereit. Nach der Auflösung des Taliban-Regimes besuchten der Vorsitzende der Übergangsregierung Hamid Karzai sowie weitere Führer des Landes im Rahmen einer offiziellen Visite im Frühjahr 2002 die russische Hauptstadt. Während dieses Besuchs unterzeichneten die beiden Seiten
17 Vereinbarungen bezüglich des Aufbaus und der Wiederherstellung der wirtschaftlichen Infrastruktur, Energieprojekte, der Lieferung von Landwirtschaftsmaschinen und Ausrüstungen, Industrieausstattung und einer Vielzahl anderer moderner Techniken.

Allerdings gelang es beiden Ländern nicht, diese Vereinbarungen effektiv zu realisieren, da die russischafghanischen Beziehungen von einer Reihe komplexer Faktoren abhängen: Erstens der Situation in Afghanistan; zweitens den Beziehungen Russlands zu führenden westlichen Staaten, im Wesentlichen den USA und drittens den sich ändernden Bedingungen in Zentralasien und der Welt allgemein.

Trotz des geringen Fortschritts in den bilateralen Beziehungen, setzt Moskau seine Unterstützung der internationalen Gemeinschaft im Kampf gegen Terrorismus und islamischem Extremismus in Afghanistan und der Region fort. Russland unterstützt die afghanische Bevölkerung zudem beim Aufbau ihrer zerstörten Wirtschaft und des Sozialbereichs. Obwohl Russland nur über eingeschränkte finanzielle Mittel zur Unterstützung Afghanistans verfügt, versuchte es einen Beitrag zu leisten. Beispielhaft für diesen Einsatz ist der Schuldenerlass Russlands gegenüber Afghanistan in Höhe von 11,5 Mrd. US-Dollar.

\section{Russische Interessen in Afghanistan}

Die russische Beteiligung am Wiederaufbau Afghanistans ist auf eine Reihe von Faktoren zurückzuführen, die mit Russlands nationalen Interessen sowie seiner wachsenden Bedeutung im globalen und regionalen System der internationalen Beziehungen in Verbindung stehen. Trotz der Vertreibung der Taliban im Jahr 2001 gehen von Afghanistan weiterhin Gefahren und Herausforderungen für die Sicherheit Russlands sowie seiner Verbündeten in Zentralasien aus. Diese Herausforderungen umschließen den Drogenhandel, islamischen Extremis- 


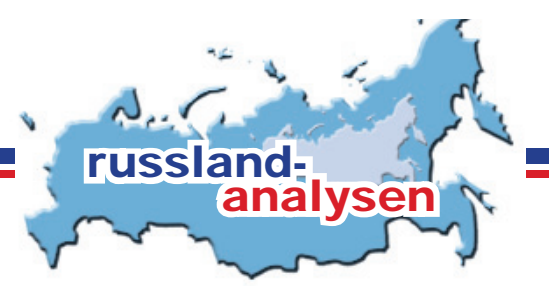

mus und Terrorismus, politische Instabilität sowie die weit verbreitete Korruption.

\section{Illegaler Drogenhandel}

Die größte Gefahr, die momentan für Russland von Afghanistan ausgeht, ist die Produktion, illegale Verbreitung sowie der Schmuggel von Drogen. Ein beachtlicher Teil der illegalen Drogen aus Afghanistan verbleibt in Russland. Entgegen der Behauptung afghanischer Behörden, die Drogenproduktion würde konstant zurückgehen (2007 lag der Umfang bei 8200 Tonnen, 2008 bei 7700 Tonnen, ein Rückgang von 6\%) bleibt die Menge des illegal nach Russland importierten afghanischen Opiums mehr oder weniger gleich. Annähernd $25 \%$ der gesamten afghanischen Drogenproduktion, diese beläuft sich auf fast vier Tonnen Opium pro Tag (genug für sechs Million Dosen reinen Heroins), gelangen von Afghanistans nördlicher Grenze über das Hoheitsgebiet der zentralasiatischen Republiken nach Russland.

Der Herointransport nach Russland erfolgt über eine Reihe von Routen - durch Tadschikistan, Usbekistan, Turkmenistan und Aserbaidschan. Ein beträchtlicher Teil des nach Russland gelangten Heroins wird nach Westeuropa weitertransportiert, der Großteil jedoch in Russland konsumiert. Laut offizieller russischer Statistik gibt es 2,5 Million Drogenabhängige im Land, was Russland den 1. Platz im weltweiten Konsum afghanischen Heroins verschafft. In den letzten Jahren hat sich die Drogensucht zu einer nationalen Krise entwickelt. Mehr als 30.000 meist junge Menschen sterben alljährlich an einer Überdosis. Das afghanische Heroin wurde somit zu einer realen Bedrohung für die Gesundheit der russischen Bevölkerung und ein ernstzunehmender Faktor bei der Verschlechterung der demographischen Situation. Folglich hat der Kampf gegen afghanische Drogen einen hohen Stellenwert in der russischen Außenpolitik.

Das afghanische Drogenproblem ist eng mit den Taliban und ihren Verbündeten verknüpft. Die Drogen stellen eine wesentliche, wenn nicht gar die hauptsächliche Einnahmequelle der Taliban dar. Die Taliban investieren in die Drogenproduktion, bieten Schutz bei der Ernte, stellen das Saatgut sowie Kredite für Drogen anbauende Bauern bereit (häufig begleitet von Gewaltanwendung), verkaufen die Drogen und sammeln die Steuern (genannt $u s h r$ ) von den Drogenproduzenten in den von ihnen kontrollierten Gebieten ein. Alles in allem belaufen sich die jährlichen Einnahmen auf fast eine halbe Milliarde Dollar, wovon die Steuern 70-75 Mio. Dollar ausmachen.

\section{Islamischer Extremismus}

Eine weitere ernsthafte Gefahr für Russland und seine Verbündeten stellt der islamische Extremismus dar, der sowohl die ideologische als auch die politische Praxis der Taliban wie auch anderer radikalislamischer Gruppen mit Verbindungen zu "Al-Qaeda« bezeichnet. Trotz der zahlenmäßigen Aufstockung der International Security Assistance Force (ISAF) sowie der USgeführten internationalen Koalitionstruppen, haben die Islamisten die Gewalt gegen die Regierung Karzai und die NATO-Truppen systematisch gesteigert und ihre Einflussgebiete ausgeweitet. Nach Angaben des Internationalen Zentrums für Sicherheit und Zusammenarbeit waren die Taliban im Jahr 2008 auf $52 \%$ des afghanischen Territoriums militärisch präsent. $\mathrm{Zu}$ Beginn des Jahres 2010 hat sich die Präsenz deutlich erhöht: Heute agieren bewaffnete Einheiten der Extremisten in $72 \%$ des Landes, inklusive der vormals ruhigen westlichen und nördlichen Provinzen, welche sich in unmittelbarer Nachbarschaft zu den zentralasiatischen Republiken befinden.

Die Taliban unterhalten enge Kontakte zu extremistischen Gruppen in Usbekistan und Tadschikistan, vor allem zur Islamischen Bewegung Usbekistans (IMU) und zur Hizb ut-Tahrir. Auf Grund der Anstrengungen der usbekischen und tadschikischen Armee und Polizei wurde ein Großteil der islamistischen Kämpfer aus diesen Ländern vertrieben. Doch haben sich die Meisten einfach der Taliban und Al-Qaeda angeschlossen, die bequeme Zufluchtsorte im benachbarten Pakistan boten. Im Frühjahr 2007 wurde ein großer Teil der IMU-Kämpfer in Pakistan von lokalen Stammesmilizen unterstützt durch die pakistanische Armee getötet. Viele agieren jedoch weiterhin, sind wieder nach Afghanistan zurückgekehrt und haben sich dort den lokalen Taliban angeschlossen. Die engen Verbindungen zwischen den islamischen Extremisten in Zentralasien und Afghanistan stellen eine direkte Gefahr für Usbekistan und Tadschikistan, aber auch für Russland dar, insofern als Moskau die zentralasiatische Region als seinen eigenen Verantwortungsbereich ansieht und sich selbst als Garant der regionalen Sicherheit versteht. Afghanische Rebellen bedrohen Russland auch, indem sie sich mit Tschetschenen verbünden, die Verbindungen zu den Resten der separatistischen Bewegungen in ihrer Heimat haben.

Die Sorge um Tschetschenien und dem übrigen Nordkaukasus ist ein Grund, warum Russland stark an einer Niederschlagung von islamischem Extremismus und Terrorismus in Afghanistan und der Region interessiert ist. Die jüngsten Selbstmordattentate in Mos- 


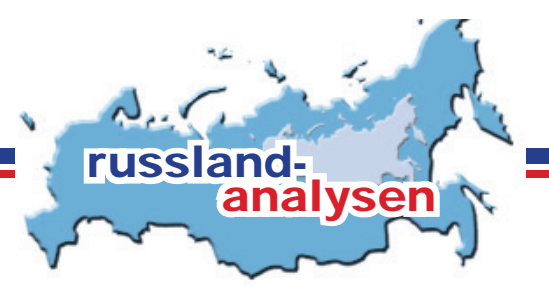

kau mit 40 zivilen Opfern illustrieren diese Gefahr allzu deutlich.

\section{Korruption \\ Weitere Sorgen Russlands beziehen sich auf die Korrup- tion in Afghanistan, welche ein solches Ausmaß ange- nommen hat, dass Afghanistan nun an zweiter Stelle hinter Somalia, dem korruptesten Staat der Welt, steht. Die Korruption zersetzt und zerstört den Wiederaufbau in Afghanistan, so dass die US-Administration von Prä- sident Obama den Kampf gegen die Korruption als vor- rangig in ihrer neuen Afghanistanstrategie erachtet. Die Korruption kann, wie auch die Drogen, zu einer grenz- überschreitenden und folglich transnationalen Erschei- nung werden. Die Korruption in Afghanistan verbrei- tet sich in der Tat und wirkt sich auf das Machtgefüge der Nachbarländer aus, inklusive einiger für Russland wichtiger Verbündeter und Partner.}

\section{Die russisch-afghanischen Beziehungen heute}

Wie oben dargestellt, steht Russland einer Reihe von ernsthaften Herausforderungen und Bedrohungen aus dem afghanischen Territorium gegenüber. Aus diesem Grund ist Russland unmittelbar daran interessiert, sich diesen Herausforderungen gemeinsam mit der afghanischen Bevölkerung und der Weltgemeinschaft zu stellen. Moskau nimmt demnach aktiv am Krieg gegen den Drogenhandel, der Auseinandersetzung mit islamischen Extremisten, dem Wiederaufbau der kriegszerstörten Wirtschaft teil und setzt sich dafür ein, dass Afghanistan zu einem friedlichen Leben zurückkehrt. Überdies kann Russland das Schicksal des afghanischen Volkes nicht einfach ignorieren, mit dem es eine lange Geschichte an guten, nachbarschaftlichen Beziehungen unterhält. Zudem sind Frieden und Stabilität in Afghanistan für eine dauerhafte und stabile Sicherheitslage in den Ländern um Afghanistan inklusive Russland notwendig.

Erst kürzlich taten sich neue Möglichkeiten für Russland auf, sich im Wiederaufbauprozess in Afghanistan zu engagieren. Unter der Schirmherrschaft der Shanghaier Organisation für Zusammenarbeit (SOZ) fand im März 2009 in Moskau eine internationale Konferenz zu Afghanistan statt. Eine der zentralen Feststellungen dieser Konferenz war, dass eine Lösung des Problems in Afghanistan im Kontext der aktuell andauernden Pattsituation zwischen den NATO-Kräften und den Taliban ohne die Beteiligung Russlands und seiner zentralasiatischen Verbündeten, auf Grund ihrer reichlichen Erfahrungen im Umgang mit Afghanistan, nicht möglich scheint. Diese Idee hat eine breite Unterstützung im
Westen inklusive der USA gefunden. Westliche Politiker und Militärs rufen Russland regelmäßig dazu auf, mit ihrer Erfahrung zu einer friedlichen Lösung der Situation in Afghanistan beizutragen. Der jüngste Appell dieser Art kam von NATO-Generalsekretär Anders Fogh Rasmussen, der bei einem Treffen mit Premierminister Putin am 17. Dezember 2009 erklärte, dass er Afghanistan als ein zentrales Thema der NATO-Russland Beziehungen betrachte.

Auf Grund seiner geopolitischen Lage und strategischen Möglichkeiten kann Russland Afghanistan auf mehreren Ebenen beeinflussen: zum Ersten dank der russisch-afghanischen bilateralen Beziehungen, die wiederholt vom russischen sowie afghanischen Präsidenten hervorgehoben werden; zum Zweiten durch die Kooperation mit seinen Verbündeten und Partnern in Zentralasien; drittens durch die Beteiligung in regionalen Organisationen wie der Shanghaier Organisation für Zusammenarbeit und der Organisation des Vertrages über kollektive Sicherheit (OVKS) sowie viertens durch die Kooperation mit internationalen Organisationen wie der UN, OSZE und NATO.

Auf bilateraler Ebene leistet Russland Afghanistan Wirtschaftshilfe in Wiederaufbauprojekten, die vormals durch die technische und wirtschaftliche Hilfe der Sowjetunion (142 Projekte) finanziert wurden, sowie geologische Erkundungen und Ausbildungen, darunter die Spezialisierung von Polizeikräften zur Drogenbekämpfung. Wenn auch begrenzt im Ausmaß, so entwickelt sich auch ein militärtechnischer Austausch zwischen den beiden Ländern. Zudem ist Russland in der multilateralen Kooperation zwischen den zentralasiatischen Republiken und Afghanistan aktiv, inklusive dem Engagement im Energiesektor. Tadschikistan ist im Moment dabei, mit finanzieller und technischer Hilfe aus Russland, das größte Wasserkraftwerk in der Region, »Songtuda«, fertig zu stellen. Der Strom, der in Songtuda produziert wird, wird nach Afghanistan und Pakistan weitergeleitet.

Die Unterstützung der NATO war eine der bemerkenswertesten Komponenten der jüngsten Afghanistanpolitik Moskaus. Russland erlaubte die Nutzung seines Luftraumes sowie von Bodeneinrichtungen zur Lieferung unterschiedlichster NATO-Güter nach Afghanistan, darunter Truppen und Waffen. Eine Vereinbarung über diese Maßnahmen wurde von Putin am Rande des NATO-Gipfels in Bukarest im April 2008 unterzeichnet. Zusätzlich signalisierte Russland, während des Treffens von Anders Fogh Rasmussen mit russischen Eliten am 17. Dezember 2009 in Moskau die Bereitschaft, die Kooperation auszuweiten. Eine solche 


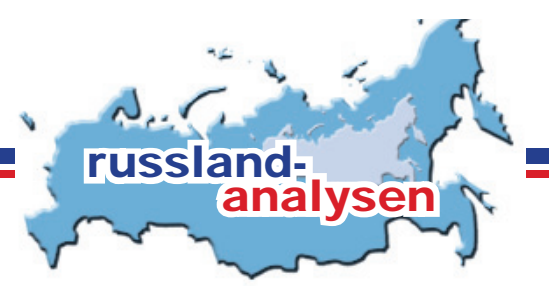

Kooperation würde auch die zentralasiatischen Republiken mit einbeziehen, deren Staatsgebiete zum Transport von NATO-Gütern verwendet werden könnten. Eine Reihe NATO-Mitgliedsstaaten haben entsprechende Vereinbarungen mit den zentralasiatischen Republiken getroffen. Es erwies sich jedoch als ein kompliziertes und langwieriges Unterfangen für den Westen, mit jeder der zentralasiatischen Republiken einzeln zu verhandeln. In Anbetracht dieser Tatsache ist der Vorschlag Russlands nur logisch, direkte Kontakte zwischen der NATO und der Organisation des Vertrages über kollektive Sicherheit, welche Russland und die zentralasiatischen Länder umfasst, einzurichten. Aus russischer Sicht sollte die Kooperation zwischen NATO und OVKS nicht allein auf den Transport von NATO-Ausrüstungen beschränkt sein, sondern einen erweiterten Dialog in Afghanistan, z. B. gemeinsame Anstrengungen bei der Drogen- und Terrorismusbekämpfung, beinhalten. Auf diesem Gebiet hat die OVKS eine Fülle an Erfahrungen.

Zusätzlich wird die Mitgliedschaft Russlands in der Shanghaier Organisation für Zusammenarbeit als eine Möglichkeit gesehen, die Situation in Afghanistan zu beeinflussen. Von Anfang an war die SOZ darauf ausgerichtet, sich für regionale Sicherheit sowie den Kampf gegen Separatismus und islamischem Extremismus einzusetzen. Die SOZ arbeitet bereits im Rahmen der SOZ-Afghanistan Kontaktgruppe mit Afghanistan zusammen, welche die beiderseitigen Anstrengungen im Kampf gegen Terrorismus und Drogenhandel koordiniert.

Die SOZ steht jedoch vor einer Reihe von Problemen, die einer Beteiligung in Afghanistan im Wege stehen. Erstens haben die Mitgliedsstaaten der SOZ eine direkte militärische Präsenz in Afghanistan abgelehnt, auch verfügt die SOZ nicht über eigene militärische Mittel. Zweitens ist ihre finanzielle Basis schwach: im letzten Jahr überstieg ihr Haushalt keine vier Million Dollar, womit gerade einmal die Personalkosten gedeckt werden könnten. Drittens verfolgt die SOZ aufgrund ihrer eingeschränkten finanziellen Mittel keine gemeinsamen wirtschaftlichen und sozialen Projekte.

Viertens gibt es eine Reihe interner Streitigkeiten zwischen SOZ-Mitgliedstaaten, darunter politische, militärische, wirtschaftliche, nationale sowie Grenzkonflikte wie auch zu weiteren Problemen. Die SOZ ist somit keine stark integrierte Organisation. Letztendlich haben die SOZ-Mitgliedstaaten unterschiedliche Ziele in und Verbindungen mit Afghanistan. Ohne Zweifel sind sie in gemeinsamen Gefahren, welche von Afghanistan ausgehen, vereint, darunter Terrorismus, nationaler Separatismus, islamischer Extremismus, Drogenhandel und allgemeine Instabilität. Jedoch werden das Ausmaß und die Priorität dieser Bedrohungen von den Mitgliedern unterschiedlich wahrgenommen. Russland ist hauptsächlich über den afghanischen Drogenhandel besorgt. Diese Sorge wird in den zentralasiatischen Republiken geteilt. Zusätzlich sind die zentralasiatischen Republiken jedoch stark über die Gefahr des islamischen Extremismus beunruhigt, der in Afghanistan entsteht. China ist stärker damit beschäftigt, seine wirtschaftlichen Interessen in Afghanistan und Zentralasien zu sichern. Gleichzeitig sind sich alle SOZ-Mitgliedstaaten des wachsenden politischen und militärischen Einflusses der USA in der Region bewusst. Dieser komplexe Block an Motiven macht die Ausarbeitung eines einheitlichen internationalen Ansatzes gegenüber Afghanistan schwierig. Dennoch sind alle an der Entwicklung von Frieden und Demokratie in Afghanistan interessiert und unterstützen aus diesem Grund die Anstrengungen der internationalen Koalition in ihrem Kampf gegen den Terrorismus in Afghanistan und Pakistan.

\section{Schluss}

Afghanistan ist, mit all seinen Problemen, aufs Neue von wichtigem außenpolitischem Interesse für Russland. Russland teilt die Ziele der internationalen Koalition in Afghanistan und unterstützt die Anstrengungen der Weltgemeinschaft, Bedrohungen seitens Afghanistans zu beseitigen. Aus diesem Grund ist Moskau, im Rahmen seiner Möglichkeiten bereit, eine aktivere Rolle beim Wiederaufbau in Afghanistan zu spielen.

Übersetzung von Christopher Laug

Über den Autor

Victor Korgun ist Professor und Vorsitzender der Abteilung für Afghanistanstudien am Institut für Orientstudien der Russischen Akademie der Wissenschaften (RAN). 


\section{Die Bedeutung Afghanistans für Russland: Regionale oder globale Strategie?}

Von Natasha Kuhrt, London

\section{Zusammenfassung}

Afghanistan ist für Russland von großer Bedeutung - vor allem mit Blick auf die eigene Sicherheit, aber auch im Rahmen einer weitergreifenden Strategie, bei der es darum geht, den Einfluss in Zentralasien zu behaupten. Russland entwickelt die Organisation des Vertrags für Kollektive Sicherheit (OVKS) als eine regionale Sicherheitsstruktur, ist jedoch momentan nicht in der Lage, einen Betrag zur Sicherheit in Afghanistan zu leisten.

Trotz Vorbehalten gegenüber der Strategie der ISAF hofft Russland jedoch, dass der NATO-Einsatz in Afghanistan erfolgreich sein wird und setzt daher die Unterstützung des ISAF-Einsatzes fort.

\section{Russland und der Westen nach dem 11. September}

Nach den Terroranschlägen des 11. September stand Russland an der Seite der USA bereit, den Terrorismus zu bekämpfen, wo immer er auftrat. Es wurde jedoch sehr bald deutlich, dass die Ziele Russlands und der USA sich deutlich unterschieden. Für Russland waren die Taliban ein Sicherheitsproblem, das es seit geraumer Zeit bekämpfte, und das eng mit den Entwicklungen und der russischen "Friedensmission" in Tadschikistan verknüpft war. Das außenpolitische Konzept Russlands aus dem Jahr 2000 unterstrich, dass die Fortsetzung des Konfliktes in Afghanistan eine reale Bedrohung für die Sicherheit der südlichen Grenze Russlands darstellte und demzufolge unmittelbar russische Interessen betraf.

In diesem Sinne war es nur logisch, dass Russland das Vorgehen der USA gegen Al-Qaeda und die Taliban begrüßte, insbesondere, da russische Entscheidungsträger seit den frühen 1990er Jahren hofften, dass die USA und Russland dem islamischen Fundamentalismus in Zentralasien gemeinsam entgegentreten würden. Entgegen den Erwartungen Russlands gaben die USA jedoch offenbar bilateralen Beziehungen zu den zentralasiatischen Staaten den Vorzug, mit dem Hauptaugenmerk auf Energie statt auf islamischem Fundamentalismus.

Russische Wissenschaftler und Entscheidungsträger kritisierten die Doktrin des "geopolitischen Pluralismus«, die Zbigniew Brzezinski formuliert hatte, und die darauf ausgerichtet war, die zentralasiatischen Staaten von Moskau so unabhängig wie möglich zu machen, und empfahl Washington bei seiner Zentralasienpolitik Partnerschaften mit der Türkei und China gegenüber der mit Russland zu bevorzugen. Darüber hinaus sollte China als Werkzeug dienen, um einem übermä-
Bigen Einfluss Russlands in der Region einen Riegel vorzuschieben.

Nachdem deutlich wurde, dass die Taliban nicht schnell eliminiert werden konnten, und die US-Präsenz in Zentralasien kein temporäres Phänomen ist, wurde Russlands Sicht auf den Militäreinsatz in Afghanistan zunehmend ambivalent. Die anfängliche Unterstützung für den Afghanistaneinsatz als »notwendigem Krieg«, im Gegensatz zu dem "gewollten Krieg« im Irak, hat abgenommen. Russland stellt nun die Legitimität des Krieges sowie seine Ausweitung in den Afghanistan-Pakis$\tan (» A f P a k »)-R a u m$ in Frage. Das Demokratisierungsprogramm der Bush-Administration in Zentralasien, das die Unterstützung "farbiger Revolutionen« im postsowjetischen Raum einschloss, wurde zu einem wesentlichen Streitpunkt zwischen den USA und Russland. Der Amtsantritt der Obama-Administration mit ihrem verqueren "Leitmotiv« eines "reset« der bilateralen Beziehungen schien der Demokratisierungsagenda, im Rahmen einer allgemeinen Abschwächung der eher normativen und verordnenden Aspekte der US-Russland-Politik, eine geringere Bedeutung zu geben. Das sollte die Bedenken Russlands bezüglich Afghanistan eigentlich besänftigen. Russland verhält sich jedoch gegenüber der Idee des »reset« zurückhaltend, betrachtet diese als beschränkt und selektiv, da sie die prioritären russischen Interessen nicht aufnimmt. Für Russland wäre nur eine Umgestaltung der Beziehungen befriedigend, die die Kooperation mit regionalen, von Russland unterstützten Sicherheitsorganisationen einschließen würde.

\section{Afghanistan in der russischen Außen- und Sicherheitspolitik}

Das außenpolitische Konzept Russlands aus dem Jahr 2008 rückt bei Sicherheitsfragen in Zentralasien die 


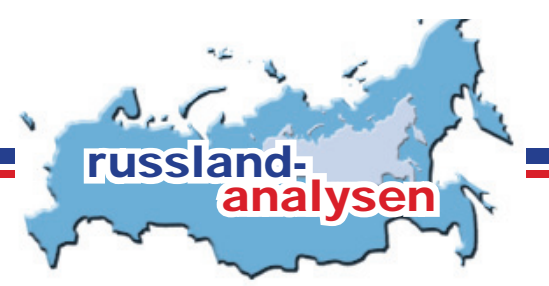

regionalen und subregionalen, von Russland unterstützten, Organisationen an erste Stelle, nämlich die Shanghaier Organisation für Zusammenarbeit (SOZ) und die Organisation des Vertrages über Kollektive Sicherheit (OVKS).

Obwohl Afghanistan im Gegensatz zu den anderen zentralasiatischen Staaten keine Energieressourcen hat, ist es dennoch ein potenziell wichtiger Transitstaat (oder ein strategisches Hinterland?). In gewisser Hinsicht liegt die Bedeutung Afghanistans jedoch eher darin, dass es eine Gefahrenquelle für die nationale Sicherheit Russlands darstellt. Afghanistan ist Drogenlieferant für die zentralasiatischen Staaten, die diese Drogen wiederum nach Russland weitertransportieren. Bis zu $90 \%$ des nach Russland importierten Heroins stammen aus Afghanistan. Russland ist der Auffassung, dass Afghanistan als wichtigster Produzent von Opium (das vor allem zur Heroinerzeugung genutzt wird) ein zentrales Sicherheitsrisiko für Russland darstellt. Alle angrenzenden zentralasiatischen Staaten sind Durchgangsländer für den Drogenhandel. Ein Großteil der gehandelten Drogen kommt aus Nordafghanistan, der Hochburg der ehemaligen von Moskau unterstützten Nordallianz.

Eine weitere Dimension, die die russische Innenpolitik betrifft, ist die Tatsache, dass viele Arbeitsmigranten aus Tadschikistan in den Handel und Vertrieb von Drogen verwickelt sind. Nur ein kleiner Teil dieser Drogen wird in den USA oder Europa, ein Großteil jedoch in Russland konsumiert. Es wird daher argumentiert, dass die USA und Europa kaum an der Eindämmung des Drogenhandels interessiert seien. Zudem erscheint die ISAF-Strategie, beim Mohnanbau »ein Auge zuzudrücken", aus Moskauer Sicht als desaströs. In der Tat wird die Grundlage der neuen NATO-Strategie, auf ehemalige Taliban zuzugehen und diese gar zu integrieren, in Moskau ablehnend aufgenommen, da befürchtet wird, diese Strategie könnte zu einem Wiederaufleben des Fundamentalismus in der Region und zu einer Destabilisierung führen, die weit über Afghanistan hinausreicht. Es wurde auch darauf hingewiesen, dass Russland besorgt ist, der wesentliche Zweck der neuen NATO-Strategie könnte die Festigung einer NATOBasis in Russlands "nahem Ausland» sein. Obwohl die Aussicht einer NATO-Mitgliedschaft Georgiens nicht mehr besteht, ist Russland in Bezug auf langfristige NATO/US-Ambitionen weiterhin erkennbar besorgt.

Afghanistan ist auch für die Zentralasienpolitik Russlands von entscheidender Bedeutung: Die sogenannte "Rückkehr Russlands nach Zentralasien" ist ein wesentliches Element des russischen Großmachtanspruchs. Der von Anatolij Tschubajs vorgeschlagene

Begriff eines "liberalen Imperialismus« ist die ideologische Grundlage dieser Strategie: Danach stellt sich Russland als eine »verantwortliche« Großmacht dar, mit der entsprechenden Verantwortung zur Gewährleistung von Sicherheit in weniger »fähigen« Staaten. Diese unlösbare Verknüpfung wurde vom Botschafter Russlands bei der NATO, Dmitrij Rogozin, hervorgehoben, der in Aufnahme des außenpolitischen Konzepts davor warnte, dass Russland genötigt wäre, mehrgleisig in die Angelegenheiten Zentralasiens zu intervenieren (kursive Hervorhebung der Autorin), wenn die NATO-Kampagne in Afghanistan scheitern sollte. Die Konsequenz eines Scheiterns wäre eine Zunahme der »drogenterroristischen Bedrohung« und ein starker Anstieg fundamentalistischer Stimmungen, die die gesamte Region destabilisieren würden. Aus diesem Grund ist die Anlage der Exitstrategie der NATO aus russischer Sicht von entscheidender Bedeutung.

\section{Regionale Sicherheit und politische Strukturen}

Die nationale Sicherheitsstrategie Russlands benennt die Unzulänglichkeiten der bestehenden globalen und regionalen Sicherheitsarchitektur, die sich im Besonderen auf die NATO als zentrale Bedrohung der internationalen Sicherheit konzentrieren. Diese Einschätzung ist unmittelbar auf die Stärkung der OVKS bezogen, die in der Nationalen Sicherheitsstrategie als ein Mittel betrachtet wird, militärischen Herausforderungen und Bedrohungen in der Region entgegenzutreten und langfristig Stabilität zu sichern. Das Außenpolitische Konzept von 2008 geht ebenfalls im Detail auf die Bedeutung der OVKS ein. Das Konzept stellt fest, dass die »internationale Autorität« der OVKS gestärkt und Verbindungen mit ähnlichen regionalen Organisationen, wie der NATO, entwickelt werden sollten. Auch lenkt es die Aufmerksamkeit auf die amerikanischen Pläne bezüglich eines "Greater Central Asia«, der Afghanistan, Pakistan und sogar Indien umfasst. Hervorgehoben wird zugleich die Bedeutung der USA und anderer westlicher Länder für die Bereitstellung moderner Technologien und anderer Modernisierungsressourcen.

Aus diesem Grunde wird ein komplexer Ansatz empfohlen, der Russland als einen Modernisierungsvermittler und - noch wichtiger - als einen Stabilitätsvermittler sieht. Eine Konsequenz wäre, dass sich Russland als ein Konkurrent im Kampf um den Großraum Zentralasien positionieren müsste.

Das außenpolitische Konzept (noch in der Amtszeit von US-Präsident Bush in Kraft getreten) schlägt vor, 


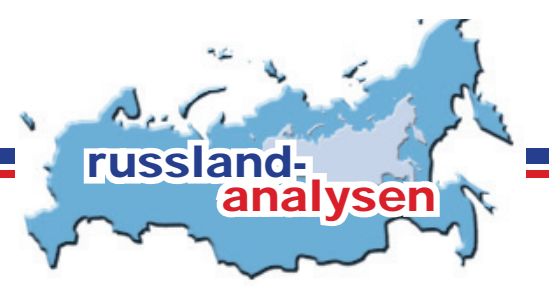

dass die OVKS die afghanisch-tadschikische Grenze gemeinsam mit der NATO schützt - ein Vorhaben, das sogar den Iran mit einbeziehen könnte. Russland betont die Erfahrung der OVKS, vor allem ihr Verständnis des Gleichgewichts zwischen den ethno-religiösen Kräften und Gruppierungen und ihren Einfluss seit den Tagen der Nordallianz. Zusätzlich wird die erneuerte Mitgliedschaft Usbekistans in der OVKS von Moskau als Profilgewinn der Organisation gewertet.

Obwohl Russland die NATO weiterhin als Bedrohung sieht, hat man die Zusammenarbeit im Fall Afghanistan fortgesetzt: Russland hat der ISAF beispielsweise sein Hoheitsgebiet für den Transit zur Verfügung gestellt, obwohl der Widerstand Russlands gegen die Toleranz der NATO gegenüber dem Mohnanbau gleichzeitig weiterhin Unbehagen erzeugt.

\section{Wichtige Akteure in der Region}

Weitere wichtige Partner Russlands in der Region sind Indien, China und der Iran. Sowohl Russland als auch China haben ihre Missbilligung gegenüber der Politik Washingtons ausgedrückt, den Iran zu isolieren. Beide Länder sehen den Iran als einen wichtigen wirtschaftlichen Partner. Auch wurde der Iran zeitweise als ein wertvoller Verbündeter gegen den Fundamentalismus in Afghanistan gesehen, obwohl die Berichte, der Iran umwerbe die Taliban, Iran zu einem unsicheren Verbündeten machten. Eine zusätzliche Komplikation ist die russische und chinesische Verurteilung nuklearer Proliferation, die es diesen beiden Ländern erschwert, Teheran zu unterstützen, solange die Streitigkeiten zwischen dem Iran und dem Westen über Teherans atomare Bestrebungen anhalten.

Indien und China sind beide sogenannte "strategische Partner« Russlands und waren bezüglich des NATO-Einsatzes in Afghanistan kritisch eingestellt, da sie über die langfristige Situation in Afghanistan nach dem Abzug der NATO-Truppen in Sorge sind. Indien ist vor allem darüber beunruhigt, dass ein NATO-Abzug zu einem Anstieg des pakistanischen Einflusses in Afghanistan sowie einem gleichzeitigen Anstieg des Fundamentalismus und zu Destabilisierung führen könnte. Die Beziehungen zwischen Indien und der NATO werden im Falle Afghanistan weiter dadurch erschwert, dass die USA neben Indien auch Pakistan an Bord zu halten sucht. Das jüngste indisch-amerikanische Nuklearabkommen schien ein besonderes Verhältnis zwischen den USA und Indien zu festigen. Indien ist jedoch weiter darüber besorgt, dass die USA seine Bedenken bezüglich dem amerikanischen Bekenntnis zur weiteren »AfPak«Strategie ignoriert.

Indien sieht, ebenso wie Russland, das Bestreben der NATO, auf die Taliban zuzugehen oder diese sogar »einzukaufen", sehr kritisch, da dies eine direkte Rückkehr der Taliban an die Macht in Afghanistan bedeuten könnte. Jede Regierung in Kabul, die sich zu sehr in Richtung Islamabad neigt, bereitet Neu-Delhi beträchtliche Sorgen. Versuche, die Idee Primakows einer russisch-indisch-chinesischen Achse wiederaufleben zu lassen, um Stabilität in Eurasien zu gewährleisten, blieben bisher reine Lippenbekenntnisse. Die Einrichtung intensiver strategischer Beziehungen zwischen den USA und Indien machen solch eine Achse dagegen weniger wahrscheinlich, obwohl russisch-indisch-chinesische Energieprojekte nicht ausgeschlossen werden können.

Russland versucht, im Rahmen der SOZ- und des BRIC-Dialogs, alternative Wege zur Lösung der Sicherheitsprobleme im zentralasiatischen Großraum zu schaffen. Im Rahmen der BRIC (Brasilien, Russland, Indien, China) versucht Russland, seinen Einfluss auf globaler Ebene zu vergrößern. Was die SOZ angeht, kann wegen der prominenten Rolle Chinas in dieser Organisation und auf Grund der bekannten Abneigung Pekings gegen jegliche Art von Militärbündnissen sowie der Bevorzugung ökonomischer Ziele gegenüber politischen und militärischen, die SOZ kaum als Mechanismus angesehen werden, mit dem ein "Scheitern« in Afghanistan bewältigt werden kann. Zudem sind die langfristigen Ziele Russlands und Chinas in der Region nicht unbedingt identisch.

\section{Die innerstaatliche und regionale Dimension}

Russlands eigene Versuche, Sicherheit zu gewährleisten, beispielsweise durch die verstärkte Sicherung der zentralasiatischen Grenzen, sind größtenteils erfolglos geblieben. Die zentralasiatischen Grenzwachen sind nach wie vor ineffizient und korrupt. Es ist fraglich, ob Russland, etwa im Rahmen der OVKS, ohne Kooperation mit der NATO den Drogenhandel eindämmen kann. In der Tat schien Außenminister Lawrow die fortgesetzte Notwendigkeit von Kooperation anzuerkennen, als er bei einem Treffen mit Hillary Clinton im Januar 2010 betonte, dass die »internationale Gemeinschaft das Potential der OVKS noch nicht vollständig nutzt, besonders deren Anstrengungen im Bereich der Drogenbekämpfung«. Im Kern würde Russland die OVKS gern an "globalen Friedenseinsätzen« beteiligen, um die Organisation auf diese Weise zu legitimieren. Im günstigsten Fall würde die NATO die OVKS als Gesprächspartner akzeptieren. Bedauerlicherweise war die NATO bisher abgeneigt, Russland solch eine Rolle einzuräu- 


\section{Analyse}

\section{Warum Russland in Afghanistan mit dem Westen kooperiert}

Von David Krickus, Washington

\section{Zusammenfassung}

Trotz der Befürchtungen, der Westen könnte die UN-mandatierte Militäroperation in Afghanistan zur Sicherung der Kontrolle über lebenswichtige Energieressourcen und Pipelines in Zentralasien ausnutzen, hat sich der Kreml dem Versuch, die Jihadisten in diesem, von Krieg zerrütteten Land zu vernichten, den USA und der NATO angeschlossen.

Eine Rückkehr der Taliban an die Macht würde den Herointransfer von Afghanistan nach Russland fördern. Die Dschihadisten würden zudem Aufstände in ganz Zentralasien vorantreiben und diese Strategie nach Russland hineintragen, wo ausländische Dschihadisten im Nordkaukasus ohnehin schon zu bewaffneten Aufständen, Terroranschlägen und Morden aufrufen. Als Konsequenz hat Russland dem US-NATOMilitärunternehmen eine gewisse Unterstützung angeboten. Beispielsweise wurde ein Luft- und Landkorridor für die Truppenversorgung im Kriegsgebiet zur Verfügung gestellt. Der Kreml hofft, nicht nur den Taliban eine Rückkehr an die Macht zu versperren, sondern auch eine aktive Rolle bei der Gestaltung der Nachkriegsordnung in Afghanistan spielen zu können. In der Zwischenzeit ermuntert er die NATO, China und anderen regionalen Mächte die Möglichkeit zu geben, zur Befriedung Afghanistans durch existierende Institutionen wie der Shanghaier Organisation für Zusammenarbeit (SOZ) und der Organisation des Vertrages über kollektive Sicherheit (OVKS) beizutragen.

\section{Die Hand reichen}

Während sich die russische Führung hartnäckig über eine NATO-Osterweiterung in Richtung ihrer Grenze beschwerte, hat sie die International Security Assistance Force (ISAF) darin unterstützt, eine Rückkehr der Taliban an die Macht in Afghanistan zu verhindern. Die Präsidenten Dmitrij Medwedew und Barack Obama versprachen auf einem Treffen in Moskau im Juli 2009, Afghanistan durch den Kampf gegen Terrorismus und Drogenhandel zu stabilisieren. Während die Allianz bewaffnete Kämpfe gegen die Dschihadisten führt, hat Russland weiterhin unter »Afghanistan -Müdigkeit» leidend - der Regierung von Hamid Karzai nichtmilitärische Hilfe angeboten. Moskau stellte Geheimdienstinformationen bereit und schulte Spezialisten zur Drogenbekämpfung, half dabei, die marode Infrastruktur des Landes wieder aufzubauen und verkaufte Waffen, Helikopter und Kommunikationsausrüstung an die afghanische Armee.

Der Transit von Waffen und nichtmilitärischem Nachschub zur ISAF via Luft- und Landkorridor durch Russland zu den Militärbasen in Afghanistan wurde zum öffentlichkeitswirksamen Beitrag Russlands zu den Bemühungen in Afghanistan. Die russische Versorgungsroute ergänzt die existierende, aber gefährdete Route des Landkorridors vom Hafen in Karatschi über den engen Khyberpass von Pakistan nach Afghanistan. Auf dieser Route haben Dschihadisten mehrfach Anschläge auf Versorgungskonvois verübt und dadurch viele Lastwagenfahrer getötet und eine beträchtliche Menge an Fracht und Fahrzeugen zerstört.
Insbesondere hat Russland den Luft- und Landkorridor als Teil des Northern Distribution Networks bereitgestellt; ca. zwei Flüge pro Tag befördern Nachschub zu den NATO-Einheiten in Afghanistan. Der Vertrag erlaubt bis zu 4,500 Flüge pro Jahr. Darüber hinaus liefert der US-Transitstützpunkt in Manas (Kirgistan) einen Großteil des Flugzeugtreibstoffes, den die US-Flugzeuge in Afghanistan verbrauchen. Weiterhin haben 30.000 US-Soldaten diesen Stützpunkt auf dem Weg in das Kriegsgebiet passiert.

Gleichzeitig wird umfangreiche Fracht über lettische Häfen durch Russland, via Termez in Kazachstan, nach Afghanistan bewegt. Schwarzmeerhäfen sind ebenfalls an dieses Netzwerk angebunden. Nach Schätzungen von US-Behörden wurde bis zum Frühjahr 2010 ungefähr ein Viertel des nichtmilitärischen Nachschubs der Allianz über diese Transportrouten befördert. Im Ergebnis konnten über 100 Mio. US-Dollar pro Jahr eingespart werden.

Russland ist bereit, wenn auch auf kommerzieller Basis und nicht unentgeltlich, die afghanische Armee mit Helikoptern, Waffen und Munition zu beliefern, welche diese für ihren Kampf gegen die Taliban einsetzen kann. Zusätzlich trainiert Russland afghanische Polizeikräfte und stellt Geheimdienstinformationen zur Verfügung, die einen Einfluss auf Militäroperationen im gesamten Kriegsgebiet haben.

\section{Die russische Politik in der Kritik}

Teile der militärischen und politischen Eliten in Russland lehnen Hilfe an die westliche Militäroperation in 
Afghanistan ab. Sie betrachten diese aus verschiedenen überzeugenden Gründen als unklug:

Die Militärintervention in Afghanistan hat den Einfluss des Westens in Zentralasien vergrößert: Die Amerikaner und Europäer wollen die zentralasiatischen Energiereserven und Pipelines, die das Öl und Gas der Region zu den internationalen Endverbrauchern transportieren, nutzen. Ihre aggressive Militäroperation könnte die russischen Anstrengungen durchkreuzen, den regionalen fossilen Energiereichtum in den wirtschaftlichen Modernisierungsprozess zu integrieren sowie dem Versuch, eine hegemoniale Stellung in ganz Eurasien wiederzuerlangen, entgegenwirken. Überlegungen dieser Art erklären, warum manch einer im Kreml die Schließung des amerikanischen Transitstützpunktes in Manas in Kirgistan befürwortet.

Eine Niederlage der Amerikaner in Afghanistan wird die russische Sicherheit erhöhen: Ein Rückschlag in Afghanistan würde die »arroganten« Amerikaner dazu zwingen, ihren schwindenden politischen und militärischen Einfluss zu akzeptieren sowie Streitigkeiten mit Russland über die NATO-Erweiterung, ein Raketenabwehrsystem und Russlands Forderung nach speziellen Einflusssphären im postsowjetischen Raum und Zentralasien beizulegen.

Ein NATO-Rückschlag wird die Aussicht auf eine neue europäische Sicherheitsarchitektur erhöhen: Sollte der NATO-Einsatz in Afghanistan scheitern, müssten die Europäer die Vergeblichkeit von Militäreinsätzen außerhalb des NATO-Gebiets anerkennen. Diese Einsicht würde sie dazu ermuntern, den Vorschlag von Präsident Dmitrij Medwedew über ein neues europäisches Sicherheitssystem anzunehmen.

Obamas Abzug aus Afghanistan ist nur eine Frage der Zeit: Obamas Afghanistanstrategie beruht darauf, gewaltige Sicherheits- und Wirtschaftsziele zu erreichen, die auf die Dauer politisch nicht nachhaltig sind. Die Taliban werden die militärische Übermacht des Westens alleine dadurch neutralisieren, indem sie direkte Konfrontationen vermeiden und darauf warten, bis der öffentliche Aufschrei, »bringt die Jungs nach Hause«, die Verantwortlichen in Amerika und Europa dazu zwingen wird, genau dies zu tun. Außerdem bedeutet die laut propagierte Entwicklungskomponente von Obamas Plan nichts anderes als das state building in einer der am meisten zurückgebliebenen Gesellschaften der Welt. Wie plausibel ist es, dass angesichts des riesigen und sich ausdehnenden US-Defizits irgendeine Regierung in Washington Milliarden von Dollar über Jahre hinweg in Afghanistan stecken wird, um diese schwierige Aufgabe zu erledigen? Warum den Amerikanern bei dieser verlorenen Sache helfen?

\section{Die Regierung befürwortet die Kooperation}

Diese Bedenken können nicht einfach von der Hand gewiesen werden, jedoch haben Premierminister Wladimir Putin und Präsident Dmitrij Medwedew die russisch-westliche Kooperation in Afghanistan auf Grund einer Reihe vitaler strategischer Gründe bekräftigt.

Festigung der Sicherheit in Russland: Afghanistan ist für viele Russen der Ursprung eines ihrer verheerendsten sozialen Probleme: die Drogenabhängigkeit. Die Vereinten Nationen geben an, dass russische Drogenabhängige fast genauso viel Heroin konsumieren wie der Verbrauch in allen EU-Ländern zusammen. Auch gibt es in Russland eine unverhältnismäßig große Zahl von Drogentoten. Der Trend steigt rapide an, Anzeichen einer Beruhigung gibt es nicht. Daher drängte Viktor Iwanow, Vorsitzender der russischen föderalen Drogenkontrollbehörde, die NATO, ein ambitioniertes Programm zur Mohnvernichtung in Afghanistan zu verabschieden. (Diese Lösung wurde von der NATO zurückgewiesen, da viele Afghanen vom Mohnanbau leben.) Bei einer erneuten Talibanherrschaft würde der Drogenhandel voraussichtlich stark ansteigen. Es ist notwendig, die Versorgungswege für Heroin von Afghanistan nach Russland zu unterbrechen. Bemerkenswert ist, dass sogar nach den verheerenden Bombenanschlägen in der Moskauer Metro die Russen laut Umfragen Drogenabhängigkeit mit $65 \%$ als größere Gefahr einstufen als den Terrorismus (60\%).

Was die politisch-militärische Bedrohung angeht, würde eine Rückkehr der Taliban an die Macht in Kabul direkte Auswirkungen auf Russland haben. Die erfolgreichen Dschihadisten würden ihre Aufmerksamkeit den 20 Millionen Muslimen in Russland zuwenden, im »Kampf um religiöse Befreiung« von der Herrschaft der "Ungläubigen«. Dies zeigt sich seit geraumer Zeit daran, dass ausländische Terroristen Waffen liefern und in Tschetschenien, Inguschetien, Dagestan und anderen Teilen des Nordkaukasus regierungsfeindliche Kämpfer trainiert haben. Im Ergebnis stieg die Zahl der Toten durch bewaffnete Auseinandersetzungen, Terrorismus und Morde in der gesamten Region. Gleichzeitig haben die Jihadisten den Kampf weit nach Russland hineingetragen. Beispiele dafür sind die St. Petersburger Zug- und die Moskauer Metroanschläge. Überdies ist in ihrer Propaganda nicht länger von nationaler Unabhängigkeit die Rede, sondern vom islamischem Dschihad. Die Kämpfer in Russland selbst meinen es ernst mit der Errichtung eines islamischen Kalifats in Russland. Auch wenn dieses Ziel ihre Möglichkeiten übersteigt, können sie ernsthafte Sicherheitsprobleme für Moskau schaffen. Sie können nicht nur einen Guerilla- 


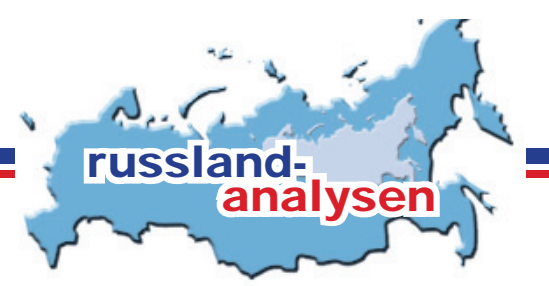

krieg führen und brutale Terroranschläge verüben, sondern auch Schienennetze, Stromkraftwerke, Energiepipelines und andere lebenswichtige Infrastruktur zerstören, während Russland gerade bemüht ist, seine Wirtschaft zu entwickeln und zu diversifizieren.

Vor diesem Hintergrund kommen russische Politiker und Experten zu dem Schluss, dass Russland seine Kooperation im Kampf gegen den islamischen Dschihadismus mit Europa und den USA "upgraden" muss, um diesem, wo immer er in Eurasien auftritt, entgegen zu wirken.

Die Sicherung Zentralasiens: Mit Verweis auf Zentralasien haben Dmitri Trenin und Alexei Malashenko festgestellt, dass »Russland ein Anwachsen des radikalen Islamismus in der gesamten Region sowie erneute Unruhen in Usbekistan und Kirgistan befürchtet. Russland hat kein ausreichendes Vertrauen in die Stabilität der zentralasiatischen Regime oder in seine eigenen Fähigkeiten, die Region vor dem Einfluss siegreicher Taliban abzuschirmen."

Das Ferganatal verbindet Usbekistan, Kirgistan und Tadschikistan und ist Heimat von ca. der Hälfte der Bevölkerung dieser drei Länder. Sollten die Taliban in Afghanistan an die Macht zurückkehren, werden sie wahrscheinlich Aufstände in diesem Gebiet fördern und die Sicherheit pro-russischer Regierungen gefährden. Es ist überflüssig hinzuzufügen, dass solch ein Chaos eine ungute Auswirkung auf die Ausbeutung und Ausfuhr lokaler fossiler Rohstoffe durch russische Firmen haben wird, die diese Ressourcen mehr oder weniger als russische betrachten. Demzufolge ist es für Russland klug, jede Art von Unterstützung zu leisten, um eine Entwicklung in Afghanistan zu fördern, die Zentralasien nicht gefährdet.

Verbindungen mit Regionalmächten und Organisationen fördern: Russland muss, neben der Kooperation mit der NATO in Afghanistan, enge Beziehungen mit den Nachbarstaaten Pakistan, Iran und China pflegen. Auch strebt Russland danach, wichtiger werdende Sicherheitsorganisationen auszubauen, vor allem die Shanghaier Organisation für Zusammenarbeit (SOZ) und die Organisation des Vertrags über kollektive Sicherheit. Der Kreml bat NATO-Generalsekretär Anders Fogh Rasmussen, die Verbindungen zwischen der NATO und den beiden Institutionen auszubauen.

Russische Verteidigungsexperten bezeichnen Pakistan als eine existenzielle Bedrohung für die Stabilität in der Region. Fedor Lukjanow, Herausgeber von Russia in Global Affairs, bemerkte, dass "die Wahrscheinlichkeit, dass in Pakistan unverantwortliche radikale Kräfte an die Macht gelangen könnten, sehr hoch ist." Obwohl "etwas Außergewöhnliches in Pakistan passieren müsse,

damit die Nuklearwaffen in jemandes Hände gelangen würden«, ist dieser Ausgang »möglich«.

Experten, die in solch nüchternen Kategorien denken, sehen mit Sorge, dass ein Sieg der Taliban in Afghanistan die Dschihadisten in Pakistan ermutigen würde. Auch wenn diese in Pakistan nicht an die Macht kommen sollten, können sie das Land destabilisieren, das Nuklearwaffen und spaltbares Material besitzt, das in die Händen von Personen gelangen könnte, die bereit wären, dieses, wenn auch vielleicht nicht gegen Gegner in Pakistan, jedoch im benachbarten Indien oder Russland einzusetzen. Die Wahrscheinlichkeit, dass dieses worst-case Szenario Realität werden könnte, mag gering sein. Es gäbe jedoch hohe Verluste an Menschenleben und als zusätzliche Folge einer solchen Tragödie wäre ein nuklearer Schlagabtausch zwischen Indien und Pakistan möglich.

Die russischen Eliten behandeln Indien traditionell mit größerem Wohlwollen als Pakistan, da letzteres eine entscheidende Rolle bei der sowjetischen Niederlage in Afghanistan spielte, während Indien im Kalten Krieg und auch heute harmonische Beziehungen mit Moskau pflegt. Der Kreml begrüßt Anstrengungen Indiens, Afghanistan im Kampf gegen die Dschihadisten zu unterstützen, die indische Truppen in Kaschmir und unschuldige Zivilisten in Mumbai getötet haben. Delhi wurde auch zu einem wichtigen Kunden russischer Militärtechnik.

Wie Russland so hat auch China eine oppositionelle muslimische Minderheit, die Uiguren in der autonomen Region Xinjiang. In ihrem Unabhängigkeitsstreben haben die Uiguren den bewaffneten Kampf aufgenommen. Gleichzeitig pflegt China umfangreiche Wirtschaftskontakte zu Zentralasien - hauptsächlich um sich einen Zugang zu fossilen Rohstoffen zu sichern - und strebt nach Afghanistans Rohstoffen und der Möglichkeit, einen Zugang zum Indischen Ozean zu erhalten.

Der Kampf gegen einen gemeinsamen Feind verbindet Peking und Moskau und ist Teil der russischen Kampagne, die mit China gemeinsame Interessen gegen einen amerikanischen Einfluss in Zentralasien und dem Fernen Osten ausbalanciert. Durch die Shanghaier Organisation für Zusammenarbeit hofft Moskau, in Zusammenarbeit mit China (sowie mit Indien und Iran) die amerikanische Hegemonie in Zentralasien, und nicht nur dort, in Frage zu stellen, während es gleichzeitig im Kampf gegen die Dschihadisten kooperiert.

Während russische Verbindungen mit den Amerikanern in Peking manche Bedenken auslösen könnten, begrüßt die Führung der Kommunistischen Partei Chinas wie auch die Regierung in Moskau, wenn auch nicht vorbehaltlos, erfolgreiche, von Amerika ange- 


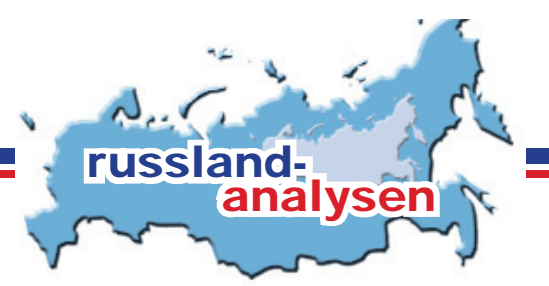

führte Anstrengungen, den Ausbau einer neuen strategischen Basis der Taliban in Afghanistan zu verhindern. Gerüchten in Washington zufolge sollen die Chinesen dem ehemaligen US-Präsidenten George W. Bush Truppen zur Unterstützung in Afghanistan angeboten haben, die dieser jedoch aus Rücksicht auf seine republikanische Basis, die solch ein Vorhaben abgeschreckt hätte, ablehnte. Zudem befürwortet die chinesische Führung Sanktionen gegen Teheran, das UNO-Forderungen zur Einstellung seines Nuklearprogrammes nicht nachkommt. Diesen Sanktionen fehlt jedoch der von US-Außenministerin Hillary Clinton bevorzugte »Biss«.

Moskau betrachtet die Beziehungen zum Iran selbstverständlich aus einer anderen und breiteren strategischen Perspektive: Neben ökonomischen Anreizen wünscht sich Russland die Aufrechterhaltung harmonischer Beziehungen mit Teheran, um sein Bild vor den eigenen Muslimen sowie jenen im Ausland zu verbessern. Kremlbeamte erinnern ihre amerikanischen Kollegen ebenfalls daran, dass der Iran eine positive Rolle dabei spielen könnte, die Rückkehr der Taliban an die Macht zu verhindern und das Nachkriegs-Afghanistan zu stabilisieren.

Neuausrichtung der Beziehungen mit dem Westen: Die russisch-amerikanische Zusammenarbeit in Afghanistan ist wohl der wichtigste aktuelle Test, inwieweit Washington und Moskau ihr Verhältnis neu "gebootet" haben. Darüber hinaus geht die russische Zusammenarbeit mit der NATO mit Medwedews Forderung nach einer neuen europäischen Sicherheitsarchitektur einher.

Beteiligung im Nachkriegs-Afghanistan: einer der Hauptgründe dafür, warum Moskau bei der Durchsetzung eines positiven Ausgangs in Afghanistan eine Rolle spielen möchte, ist die Erwartung, bei der Gestaltung der Nachkriegsarchitektur mit zu entscheiden. Wenn Russland am Rande stehen würde, müsste es jedes Ergebnis akzeptieren, dass die siegreiche Koalition bestimmen würde.

Während es also einige russische Strategen geben wird, die ihre Herren anstiften wollen, in Afghanistan Schaden anzurichten, und nicht mit der Allianz

zu kooperieren, kalkulieren jene, die eine Kooperation bevorzugen, weit umsichtiger.

\section{Zusammenfassung}

Letzten Endes können russische Sicherheitsexperten folgende zwingende Tatsache nicht vergessen: amerikanische und NATO-Truppen bekämpfen eben jene Dschihadisten, die die Nachfrage russischer Drogenabhängiger bedienen, die den Aufständen im Nordkaukasus bewaffnete Unterstützung leisten und die pro-russische Regime in Zentralasien bedrohen. Insbesondere Zentralasien stellt sowohl ein enormes Operationsgebiet für dschihadistische Interventionen wie auch einen direkten Weg nach Russland dar und bedroht die energieorientierte wirtschaftliche Entwicklungsstrategie des Kreml. Der Ausgang des Kampfes in Afghanistan wird daher mit Sicherheit einen Einfluss auf die Zukunft in Eurasien haben.

Vor diesem Hintergrund wird deutlich, dass Russland eine Reihe gewichtiger Gründe hat, die ISAF-Truppen zu unterstützen, welche bemüht sind, eine Rückkehr der Taliban in Afghanistan zu verhindern. Daher hat Russland, als Teil des Northern Distribution Network, Luft- und Landkorridore zur Verfügung gestellt. Der Kreml rechnet damit, dass diese Kooperation ihm einen Sitz am Verhandlungstisch nach dem Krieg verschafft und er somit das Schicksal Afghanistans sowie das der gesamten Region mitbestimmen kann.

Der Bericht des russischen Außenministers Lawrow, der eine Wende in Richtung Westen befürwortet, geht mit der Moskauer ISAF-Unterstützung einher. Besonnenheit verlangt jedoch, dass sich russische Strategen auch um die Antwort einer unbequemen Frage kümmern: Was muss Moskau unternehmen, sollte Obamas Plan, eine Rückkehr der Taliban an die afghanische Macht zu verhindern, scheitern? Dieses Ergebnis würde Russland und zu einem geringeren Teil auch die NATO mit einem monumentalen Sicherheitsproblem konfrontieren.

Übersetzung: Christoph Laug

Über den Autor

Dick Krickus ist emeritierter Distinguished Professor an der University of Mary in Washington und hatte den H.L.-Oppenheimer-Lehrstuhl für Strategie der Kriegsführung an der Universität des US Marine Corps inne. Seine letzte Monographie, Medvedev's Plan: Providing Russia With a Voice But Not a Veto in a New European Security System, wurde vom U.S. Army War College im Dezember 2009 herausgegeben.

Lesetipps

- Dmitri Trenin and Alexey Malashenko, "Afghanistan: A View from Moscow”, Carnegie Endowment for International Peace, 2010.

- Andrew C. Kuchins and Thomas M. Sanderson, "The Northern Distribution Network and Afghanistan," Center For Strategic and International Studies, January 10, 2010.

- Samuel Charap, "Assessing the 'Reset' and the Next Steps for U.S. Russia Policy," Center for American Progress, April 2010. 


\section{Dokumentation}

\section{UNO-Schätzungen über Produktion und Konsum von Opium und Heroin}

Regionale Verteilung des weltweiten Heroinkonsums 2008 (Gesamtkonsum 340 t)

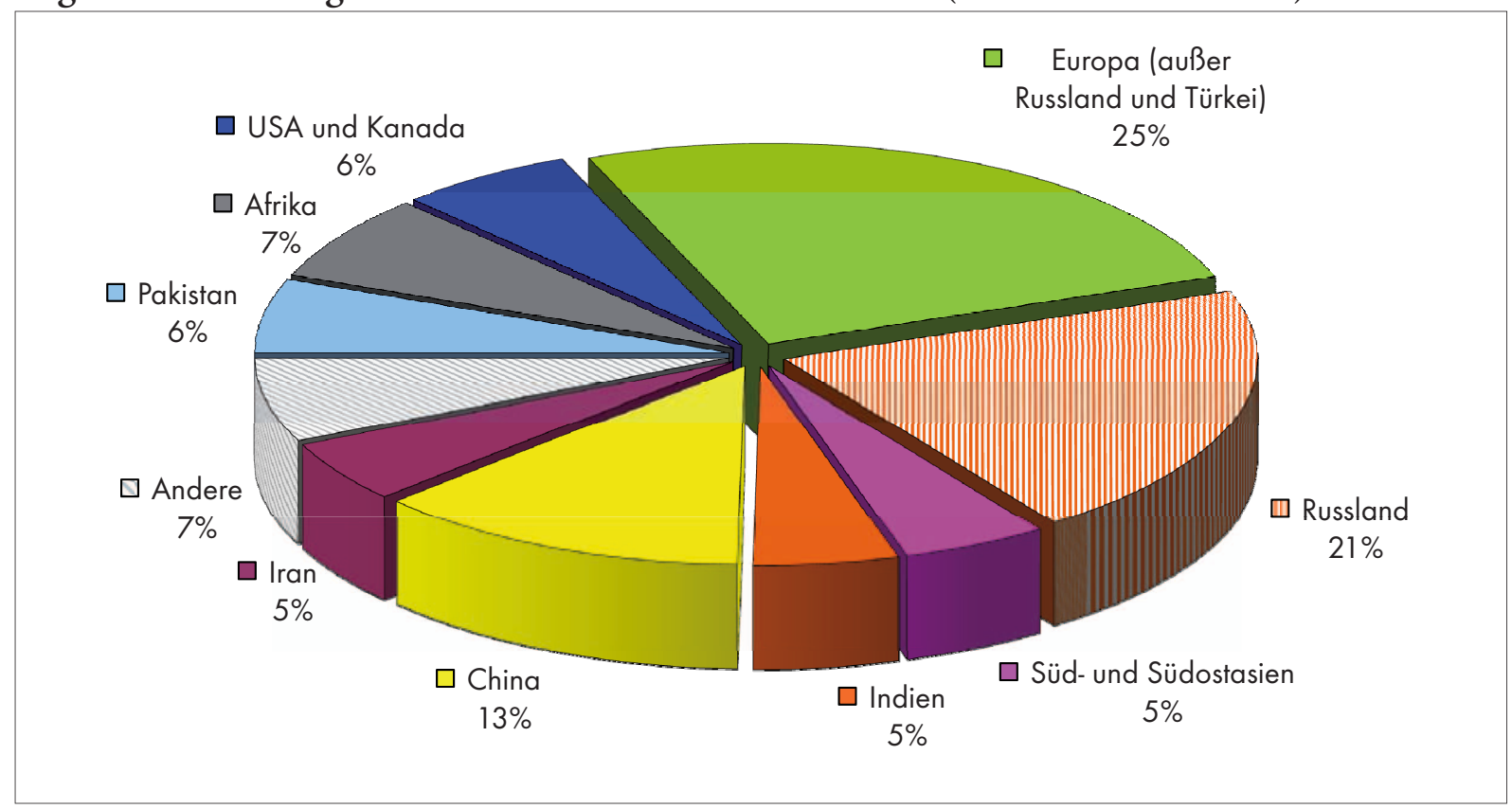

Regionale Verteilung des weltweiten Opiumkonsums 2008 (Gesamtkonsum 1.100 t)

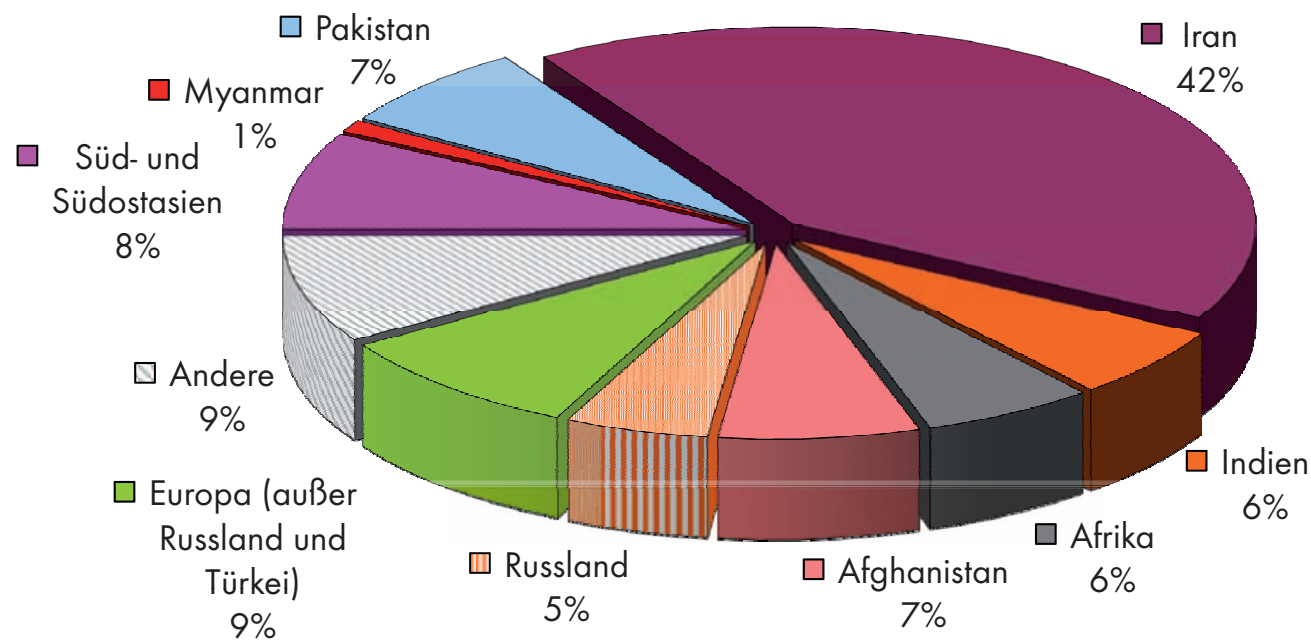

Quelle: Addiction, Crime and Insurgency. The transnational threat of Afghan opium, United Nations Office on Drugs and Crime (UNODC), October 2009, S. 11, 27,

hitp://www.unodc.org/documents/data-and-analysis/Afghanistan/Afghan_Opium_Trade_2009_web.pdf 
Opium: Anbauflächen 1994-2008 (in Hektar)

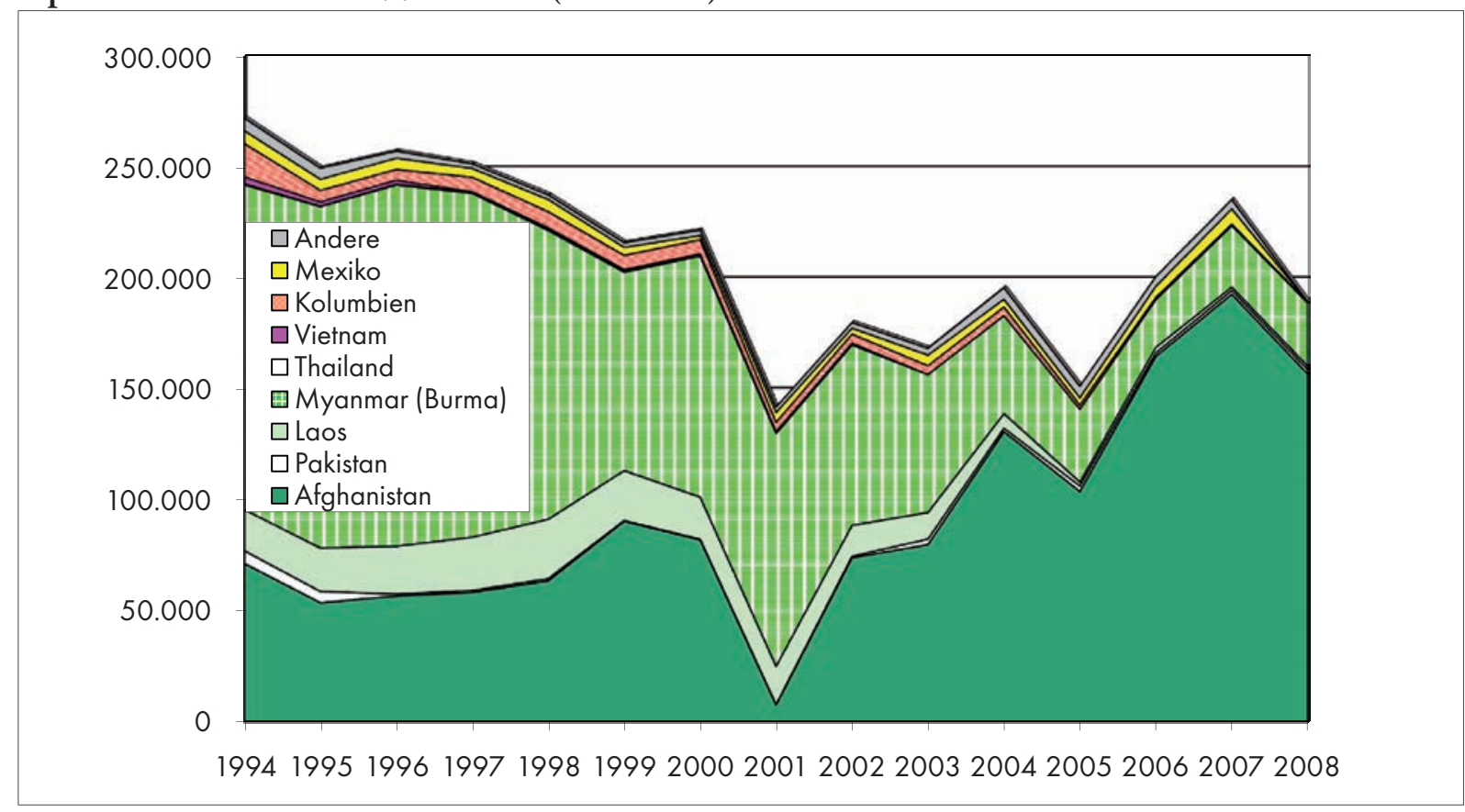

Opium: Geschätzte Erzeugung 1994-2008 (in t)

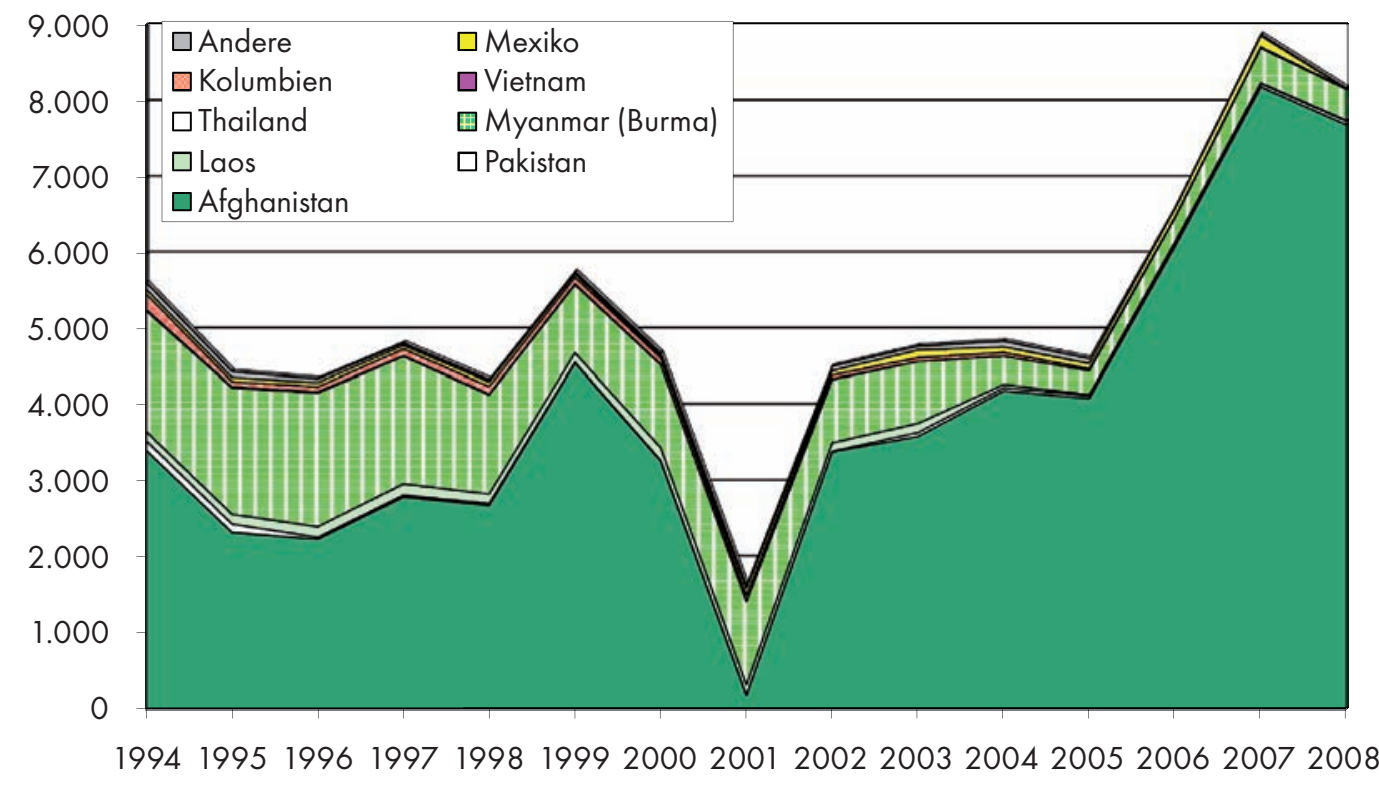

Quelle: United Nations Office on Drugs and Crime Vienna: World Drug Report 2009, New York: United Nations 2009, S. 34 , http://www.unodc.org/documents/wdr/WDR_2009/WDR2009_eng_web.pdf 


\section{Chronik}

\section{Der sowjetische Afghanistankrieg 1979-1989}

Die folgende Chronik wurde auf Basis russischer Internetquellen zusammengestellt. Sie erhebt keinen Anspruch auf Vollständigkeit und gibt bewusst eine russische Sichtweise wieder.

\begin{tabular}{|c|c|c|}
\hline \multicolumn{2}{|c|}{ Datum } & Ereignis \\
\hline \multirow[t]{9}{*}{1979} & 16.3 .1979 & $\begin{array}{l}\text { Verteidigungsminister Dmitrij Ustinow befiehlt dem Generalstab, eine Luftlandedivision und drei } \\
\text { Regimenter der Luftstreitkräfte zur möglichen Verlegung nach Afghanistan vorzubereiten. }\end{array}$ \\
\hline & 17.-19.3.1979 & $\begin{array}{l}\text { Auf der Sitzung des Politbüros der KPdSU werden die Lage in Afghanistan erörtert und die Ersu- } \\
\text { chen der afghanischen Führung, sowjetische Truppen einzusetzen. }\end{array}$ \\
\hline & 1.7.1979 & $\begin{array}{l}\text { Verlegung des 111. Luftlandebataillons nach Bagram zum Schutz der sowjetischen Transportflug- } \\
\text { zeuge. Das sind die ersten regulären sowjetischen Truppen in Afghanistan. Berater und Spezialkräfte } \\
\text { sind bereits seit längerem im Einsatz. }\end{array}$ \\
\hline & 14.9.1979 & $\begin{array}{l}\text { Konflikte in der Spitze der Demokratischen Volkspartei Afghanistans. Nur Muhammad Taraki wird } \\
\text { ermordet. An seine Stelle tritt Hafizullah Amin. }\end{array}$ \\
\hline & Dezember & Die Mudschahidin werden auf 40.000 Mann geschätzt. Sie sind in 12 Provinzen aktiv. \\
\hline & 8.12 .1979 & $\begin{array}{l}\text { Auf einer Beratung, an der Breschnew, Andropow, Gromyko und Suslow teilnehmen, wird die Lage } \\
\text { in Afghanistan erörtert. Es wird entschieden, Amin durch Einsatz von Spezialkräften zu beseitigen } \\
\text { und Babrak Karmal an seine Stelle zu setzen. }\end{array}$ \\
\hline & 12.12.1979 & Das Politbüro der KPdSU beschließt sowjetische Truppen nach Afghanistan zu senden. \\
\hline & 25.12.1979 & $\begin{array}{l}\text { Die ersten sowjetischen Truppen (108. MotSchützendivision, 103. Gardeluftlandedivision) über- } \\
\text { schreiten die Grenze. }\end{array}$ \\
\hline & 27.12.1979 & $\begin{array}{l}\text { Operation »Schtorm 33«. Der Palast Amins wird gestürmt. Karmal übernimmt die Macht in } \\
\text { Afghanistan. }\end{array}$ \\
\hline \multirow[t]{11}{*}{1980} & Januar & $\begin{array}{l}\text { Die sowjetischen Streitkräfte besetzen Schlüsselpositionen. } 21 \text { Provinzzentren und } 9 \text { Flugplätze sind } \\
\text { in ihrer Hand. Sie umfassen 75.000 Mann. }\end{array}$ \\
\hline & 7.1.1980 & $\begin{array}{l}\text { Erste Operation ist die Deblockierung von Nahrin (Provinz Baghlan), wo es am 1. Januar zum Auf- } \\
\text { stand eines afghanischen Artillerieregiments gekommen war. }\end{array}$ \\
\hline & 14.1.1980 & $\begin{array}{l}\text { Die Mehrheit der Vollversammlung der UN verurteilt den Einmarsch sowjetischer Truppen in } \\
\text { Afghanistan. }\end{array}$ \\
\hline & Februar & $\begin{array}{l}\text { Die »Duschmanen« (sowjetische Bezeichnung für die Mudschahidin) greifen in der Nähe Kabuls } \\
\text { eine Patrouille an. } 12 \text { sowjetische Soldaten werden getötet. Die sowjetische Botschaft in Kabul wird } \\
\text { beschossen, mehrere Botschaftsangehörige werden getötet. }\end{array}$ \\
\hline & 23.2 .1980 & Bei einem Verkehrsstau im Salang-Tunnel sterben 16 Soldaten durch Autoabgase. \\
\hline & & Operation »Udar» in den Zentralprovinzen \\
\hline & März & Erste große Angriffsoperation gegen die Mudschahidin: Kurnar-Offensive \\
\hline & 11.5 .1980 & Bei Gefechten in der Provinz Kunar erleidet ein Bataillon der Spezialkräfte schwere Verluste. \\
\hline & & Beginn des Baus von Stützpunkten und Sicherungsstellungen entlang der wichtigsten Verkehrswege. \\
\hline & & Kampfhandlungen in neun Provinzen \\
\hline & 3.8.1980 & $\begin{array}{l}\text { Das Aufklärungsbataillon der 201. MotSchützendivision gerät in einen Hinterhalt. } 45 \text { Soldaten wer- } \\
\text { den getötet. }\end{array}$ \\
\hline
\end{tabular}




\section{Der sowjetische Afghanistankrieg 1979-1989 (Fortsetzung)}

\begin{tabular}{|c|c|c|}
\hline \multicolumn{2}{|c|}{ Datum } & Ereignis \\
\hline \multirow{5}{*}{1981} & & Desertionen in der afghanischen Armee nehmen Massencharakter an. \\
\hline & September & $\begin{array}{l}\text { Operation »Marmol« in Masar-e Scharif. Zerschlagung einer Basis der Mudschahidin } 30 \text { km süd- } \\
\text { lich von Masar-e Scharif. }\end{array}$ \\
\hline & $\mathrm{ab} 6.9 .1981$ & 4. Pandshir-Operation (»Kan'on«) \\
\hline & $\begin{array}{l}\text { Oktober- } \\
\text { Dezember } \\
1981 \\
\end{array}$ & Hepatitisepidemie in der 40. Armee \\
\hline & Dezember & Operation zur Zerschlagung einer Basis der Mudschahidin in Darzab. \\
\hline \multirow[t]{6}{*}{1982} & Januar & Gefechte in Kandahar \\
\hline & 5.4 .1982 & $\begin{array}{l}\text { Sowjetische Truppen überschreiten bei Kampfhandlungen die iranische Grenze. Die iranischen Luft- } \\
\text { streitkräfte schießen zwei sowjetische Hubschrauber ab. }\end{array}$ \\
\hline & Mai-Juni & Operation im Pandschir-Tal, an der 12.000 Soldaten und 320 Panzerfahrzeuge teilnehmen. \\
\hline & Juli & Von 286 Bezirken Afghanistans sind 50 unter voller Kontrolle der Regierung, 156 teilweise. \\
\hline & 3.11 .1982 & $\begin{array}{l}\text { Beim Zusammentreffe zweier Kolonnen im Salang-Tunnel ersticken } 64 \text { sowjetische und } 112 \text { afgha- } \\
\text { nische Soldaten durch Autoabgase. }\end{array}$ \\
\hline & 10.11 .1982 & Tod Leonid Breschnews \\
\hline \multirow[t]{3}{*}{1983} & April & $\begin{array}{l}\text { Operation zur Zerschlagung von Mudschahidin-Abteilungen in der Provinz Kapis. } 14 \text { sowjetische } \\
\text { Soldaten werden getötet, } 63 \text { verwundet. }\end{array}$ \\
\hline & Juli & Angriff der Mudschahidin auf Chost \\
\hline & Winter & $\begin{array}{l}\text { Intensivierung der Kämpfe. Erstmals führen die Mudschahidin die Kampfhandlungen den Win- } \\
\text { ter über fort. }\end{array}$ \\
\hline \multirow[t]{4}{*}{1984} & 16.1.1984 & $\begin{array}{l}\text { Die Mudschahidin schießen ein Kampfflugzeug des Typs Su-25 ab. Das ist der erste erfolgreiche Ein- } \\
\text { satz einer "Stinger». }\end{array}$ \\
\hline & 21.4 .1984 & $\begin{array}{l}\text { Bei Operationen im Pandschir-Tal gerät ein MotSchützenbataillon in einen Hinterhalt. } 53 \text { Soldaten } \\
\text { werden getötet, } 58 \text { verletzt. }\end{array}$ \\
\hline & Dezember & Operation zur Zerschlagung einer Basis der Mudschahidin in den Bergen der Provinz Farach. \\
\hline & & 1984 werden insgesamt 17 Flugzeuge und 49 Hubschrauber abgeschossen. \\
\hline \multirow[t]{6}{*}{1985} & 21.4 .1985 & $\begin{array}{l}\text { „Drama von Marawara«. In der Nähe der pakistanischen Grenze werden } 28 \text { Aufklärer der Spezial- } \\
\text { kräfte getötet. }\end{array}$ \\
\hline & 26.4 .1985 & $\begin{array}{l}\text { Aufstand von } 24 \text { sowjetischen und afghanischen Kriegsgefangenen, die in Badabera gefangen gehal- } \\
\text { ten werden. Alle werden getötet. }\end{array}$ \\
\hline & 25.5 .1985 & $\begin{array}{l}\text { Operation in Kunar. Die } 4 \text {. Kompanie des } 149 \text {. GardeMotSchützenregiments wird eingekesselt. } 23 \\
\text { Soldaten werden getötet, } 28 \text { verletzt. }\end{array}$ \\
\hline & $\begin{array}{l}\text { Mitte des } \\
\text { Jahres }\end{array}$ & Generalsekretär Gorbatschow beauftragt das Politbüro, die Afghanistanpolitik neu zu durchdenken. \\
\hline & Juni & Armeeoperation im Pandschir-Tal \\
\hline & & $\begin{array}{l}\text { Das »Besondere Kontingent sowjetischer Streitkräfte in Afghanistan« (40. Armee) erreicht zahlenmä- } \\
\text { Big seine maximale Stärke während des Krieges. Es umfasst in diesem Jahr 108,800 Mann. }\end{array}$ \\
\hline
\end{tabular}




\section{Der sowjetische Afghanistankrieg 1979-1989 (Fortsetzung)}

\begin{tabular}{|c|c|c|}
\hline \multicolumn{2}{|c|}{ Datum } & Ereignis \\
\hline \multirow[t]{10}{*}{1986} & Februar & $\begin{array}{l}\text { Auf dem XXVII. Parteitag der KPdSU teilt Gorbatschow mit, dass ein Plan zum etappenweisen } \\
\text { Abzug der Streitkräfte ausgearbeitet wird. }\end{array}$ \\
\hline & Februar & $\begin{array}{l}\text { Operation gegen die Gruppierung von A Basir. Zerschlagung einer Basis bei Marulgad in der Pro- } \\
\text { vinz Nangarhar. }\end{array}$ \\
\hline & März & Kampfhandlungen in der Provinz Paktia. Neuerliche Operation gegen Marulgad (Nangahar). \\
\hline & 4.-20.4.1986 & Operation zur Zerschlagung der Basis Dzhabar. Niederlage der Mudschahidin. \\
\hline & 4.5 .1986 & Das Plenum des ZK der afghanischen Partei beschließt einen Kurs der nationalen Versöhnung. \\
\hline & 16.6 .1986 & $\begin{array}{l}\text { Operation »Manevr« in der Provinz Tachar. Bei Kämpfen des } 783 \text {. Aufklärungsbataillons (Kundus) } \\
\text { mit der Abteilung von Kazi Kabir werden } 18 \text { Soldaten getötet, } 22 \text { verwundet. }\end{array}$ \\
\hline & 15.7 .1986 & Die 40. Armee umfasst 133 Bataillone. 82 von ihnen sind für Sicherungsaufgaben eingesetzt. \\
\hline & $\begin{array}{l}18 .- \\
26.8 .1986\end{array}$ & $\begin{array}{l}\text { Angriff auf die Festungsregion Kokari-Sharshari (Provinz Herat). Operation "Sapadnja» unter Lei- } \\
\text { tung von Armeegeneral Valentin Warennikow. }\end{array}$ \\
\hline & Herbst & Die Aufklärungsgruppe von Major Below erbeutet in der Region Kandahar drei Stinger-Raketen. \\
\hline & $\begin{array}{l}\text { 15.- } \\
30.10 .1986\end{array}$ & Abzug von sechs Regimentern der 40. Armee (8.000 Mann). \\
\hline \multirow[t]{9}{*}{1987} & 15.1.1987 & Beginn der Politik der nationalen Versöhnung \\
\hline & $\begin{array}{l}\text { 16.1.-21.2. } \\
1987\end{array}$ & Operation »Udar» (Provinz Kundus) \\
\hline & $\begin{array}{l}4.2 .- \\
11.3 .1987 \\
\end{array}$ & Operation »Schkwal« (Provinz Kandahar) \\
\hline & März & Operation »Grosa» (Provinz Gazni) \\
\hline & März & Operation "Krug« in den Provinzen Kabul und Logar. \\
\hline & $\begin{array}{l}12 .- \\
24.4 .1987\end{array}$ & Operation »Wesna« (Provinz Kabul) \\
\hline & Mai & Operation »Zalp« in den Provinzen Logar, Paktia, Kabul. Operation »ug- $87 «$ in der Provinz Kandahar. \\
\hline & 1.6.1987 & Generalleutnant B. Gromow wird Befehlshaber der 40. Armee. \\
\hline & 23.11 .1987 & Beginn der Operation »Magistral» zur Deblockierung der Stadt Host. \\
\hline \multirow[t]{5}{*}{1988} & Januar & Abschluss der Operation »Magistrale» \\
\hline & 9.2 .1988 & $\begin{array}{l}\text { In der "Prawda« teilt Gorbatschow mit, dass die UdSSR ihre Streitkräfte aus Afghanistan abziehen } \\
\text { wird und in welchem Zeitrahmen dies geschehen soll. }\end{array}$ \\
\hline & 15.5.1988 & $60 \%$ des Territoriums von Afghanistan wird von den Mudschahidin kontrolliert. \\
\hline & $\begin{array}{l}15.5 .- \\
1.8 .1988\end{array}$ & $\begin{array}{l}\text { Die sowjetischen Truppen ziehen sich aus den Garnisonen Dschalalabad, Ghazni, Gardez, Kanda- } \\
\text { har, Lashkar Gah, Faisabad und Kundus zurück. }\end{array}$ \\
\hline & 7.8.1988 & Die afghanischen Regierungstruppen geben Kundus widerstandslos auf. \\
\hline \multirow[t]{3}{*}{1989} & 23.-26.1.89 & Operation »Tajfun«. Die letzte Operation der sowjetischen Truppen in Afghanistan. \\
\hline & 4.2 .1989 & Die letzten sowjetischen Truppen verlassen Kabul \\
\hline & 15.2 .1989 & $\begin{array}{l}\text { Die sowjetischen Truppen verlassen Afghanistan. Als letzter überschreitet Generalleutnant B. Gromow } \\
\text { die Brücke über den Grenzfluss Amudarja. }\end{array}$ \\
\hline
\end{tabular}

Zusammengestellt nach russischen Internetquellen: http://ru.wikipedia.org/wiki/\%D0\%90\%D1 \%84\%D0 \%B3 \%D0 \%BO \%D0 \%BD \% D1 \%81 \%DO \%BA \%D0 \%BO \%Dl \%8F_\%DO \%B2 \%D0 \%BE \%DO \%B9 \%DO \%BD \%D0 \%BO_\%281979\%E2 \%80\%941989\%29; http:// www. $101 \mathrm{msp} . r$ index.php?option=com_content\&view=article\&id=38\&ltemid $=31$ 


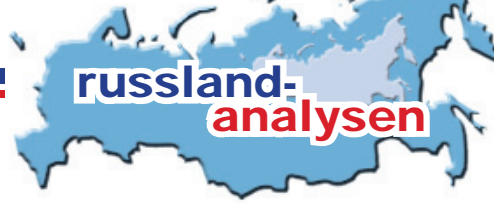

\section{Dokumentation}

\section{Sowjetische Verluste in Afghanistan 1979-1989}

\begin{tabular}{|l|c|c|c|c|c|c|}
\hline \multirow{2}{*}{} & \multicolumn{3}{|c|}{ Gesamtverluste } & \multicolumn{3}{c|}{ Im Kampf gefallen: } \\
\cline { 2 - 7 } & Insgesamt & Offiziere & $\begin{array}{c}\text { Unteroffiziere u. } \\
\text { Mannschaften }\end{array}$ & Insgesamt & Offiziere & $\begin{array}{c}\text { Unteroffiziere u. } \\
\text { Mannschaften }\end{array}$ \\
1979 & 86 & 10 & 76 & 70 & 9 & 61 \\
1980 & 1.484 & 199 & 1.285 & 1.229 & 170 & 1.059 \\
1982 & 1.298 & 189 & 1.109 & 1.033 & 155 & 878 \\
1983 & 1.948 & 238 & 1.710 & 1.623 & 215 & 1.408 \\
1984 & 1.446 & 210 & 1.236 & 1.057 & 179 & 878 \\
1985 & 2.343 & 305 & 2.038 & 2.060 & 285 & 1.775 \\
1986 & 1.868 & 273 & 1.595 & 1.552 & 240 & 1.312 \\
1987 & 1.333 & 216 & 1.117 & 1.068 & 198 & 870 \\
1988 & 1.215 & 212 & 1.003 & 1.004 & 189 & 815 \\
1989 & 759 & 117 & 642 & 639 & 106 & 533 \\
Summe (errechnet) & 13.833 & 1.979 & 11.854 & 11.381 & 9 & 37 \\
Summe (angegeben) & 13.747 & 1.969 & 11.778 & 11.311 & 1.746 & 9.565 \\
\hline
\end{tabular}

Quelle: Prawda 17.8.1989, S. 6.; 1999 wurden leicht korrigierte Zahlen veröffentlicht. Nun wurden die Verluste mit 15.051 angegeben (Sowjetische Streitkräfte 14.427, KGB 576, Innenministerium 28). Todesfälle durch Krankheit und Soldaten, die im Hospital ihren Verletzungen erlegen sind, sind in diesen Angaben wahrscheinlich nicht enthalten.

Für die afghanische Bevölkerung war der sowjetische Einmarsch eine Katastrophe. Die Schätzungen über die Zahl der afghanischen Kriegstoten schwanken zwischen 100.000 und einer Million. 3 Millionen wurden verwundet, 1,2 Millionen erlitten bleibende Behinderungen. Etwa 2 Millionen Afghanen fü̈chteten aus ihrer Heimatregion in andere Regionen des Landes. Weitere 5 Millionen gingen über die Grenze nach Pakistan und in den Iran.

\section{Verteilung der sowjetischen Verluste nach Nationalitäten 1979-1989}

\begin{tabular}{|l|c|c|}
\hline & absolut & in $\%$ \\
Insgesamt & 13.833 & $\mathbf{1 0 0 , 0 0 \%}$ \\
Russen & 6.879 & $49,73 \%$ \\
Ukrainer & 2.374 & $17,16 \%$ \\
\hline Usbeken & 1.067 & $7,71 \%$ \\
Belorussen & 611 & $4,42 \%$ \\
Kasachen & 361 & $2,61 \%$ \\
Turkmenen & 281 & $2,03 \%$ \\
Tadschiken & 239 & $1,73 \%$ \\
Moldawier & 195 & $1,41 \%$ \\
Aserbaidschaner & 195 & $1,41 \%$ \\
Kirgisen & 102 & $0,74 \%$ \\
Armenier & 98 & $0,71 \%$ \\
Georgier & 81 & $0,59 \%$ \\
Litauer & 57 & $0,41 \%$ \\
Letten & 23 & $0,17 \%$ \\
Esten & 15 & $0,11 \%$ \\
Andere & 1.255 & $9,07 \%$ \\
\hline
\end{tabular}

Quelle: Krasnaja Swesda 15.2.1990, S. 4 


\section{Umfrage}

\section{Internationale Umfrage zum NATO-Einsatz in Afghanistan, Juli 2009}

Die UNO hat eine NATO-Mission in Afghanistan autorisiert. Was halten Sie von dieser Mission?

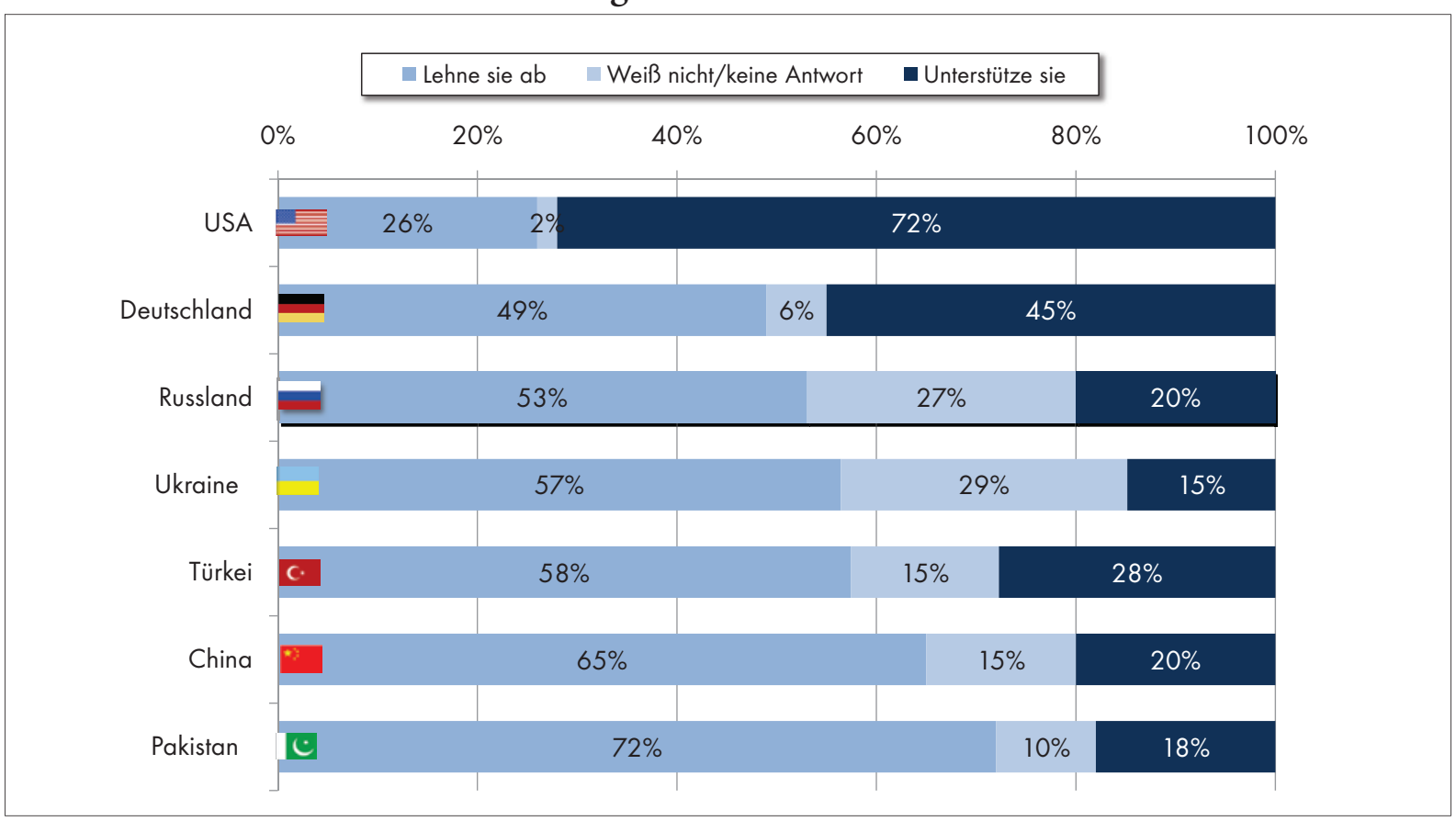

Soll die NATO-Mission in Afghanistan fortgeführt werden?

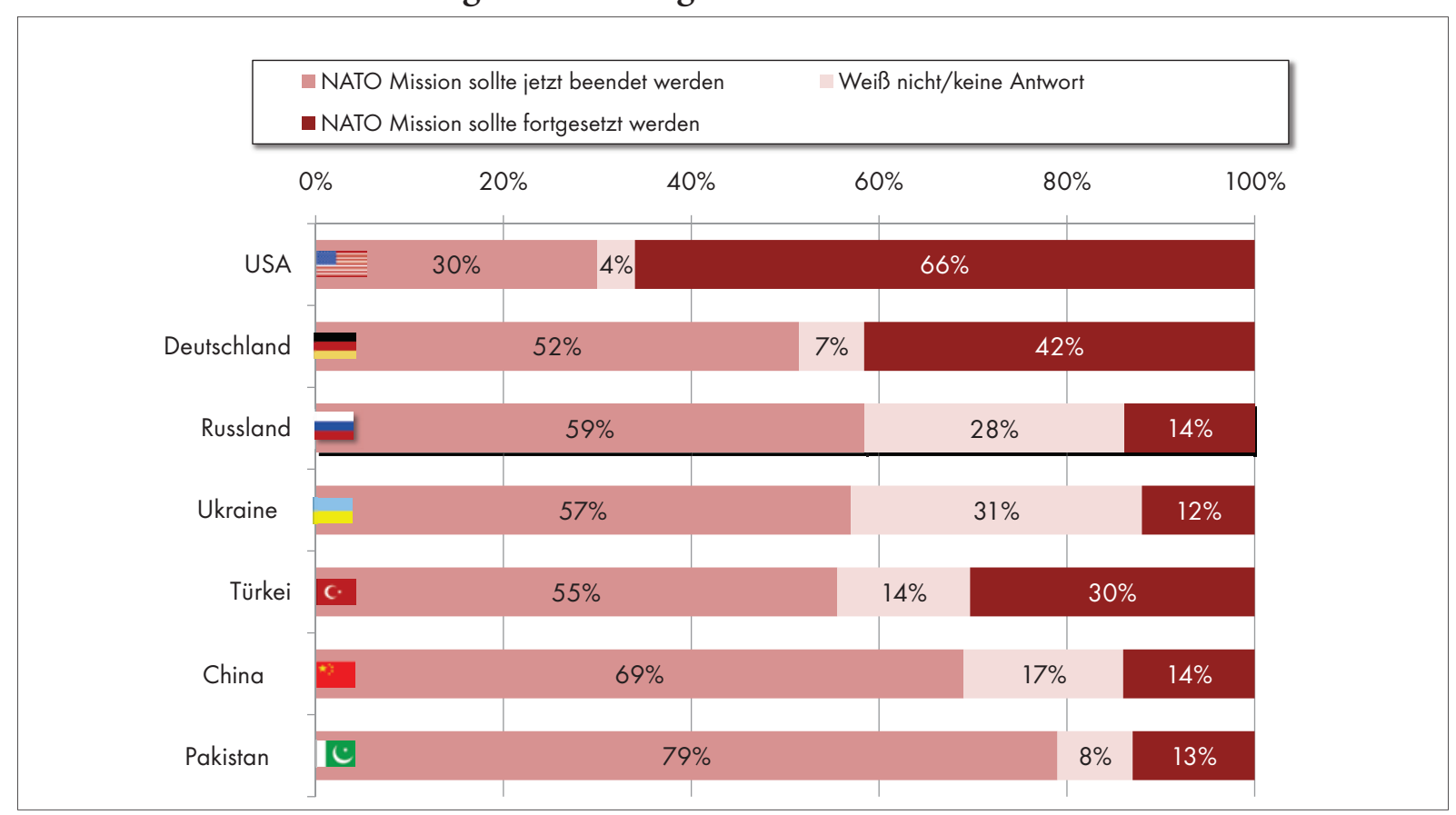

Quelle: Umfragen von WorldPublicOpinion vom Juli 2009,

http://www.worldpublicopinion.org/pipa/pdf/jul09/WPO_Afghan_Jul09_quaire.pdf 
Glauben Sie, dass die Bevölkerung Afghanistans will, dass die NATO-Truppen bleiben?

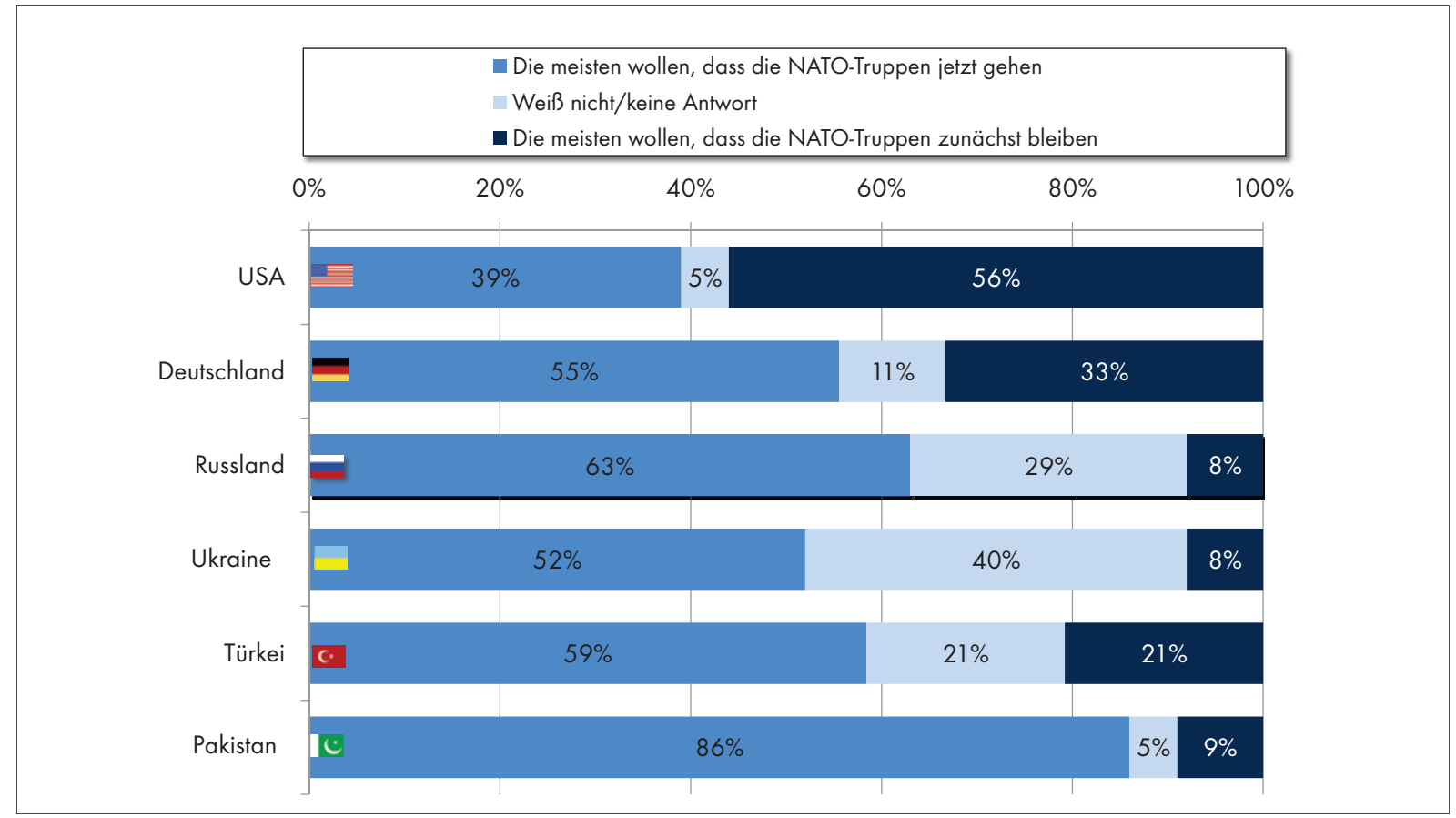

Wie bewerten Sie eine mögliche Rückkehr der Taliban an die Macht in Afghanistan?

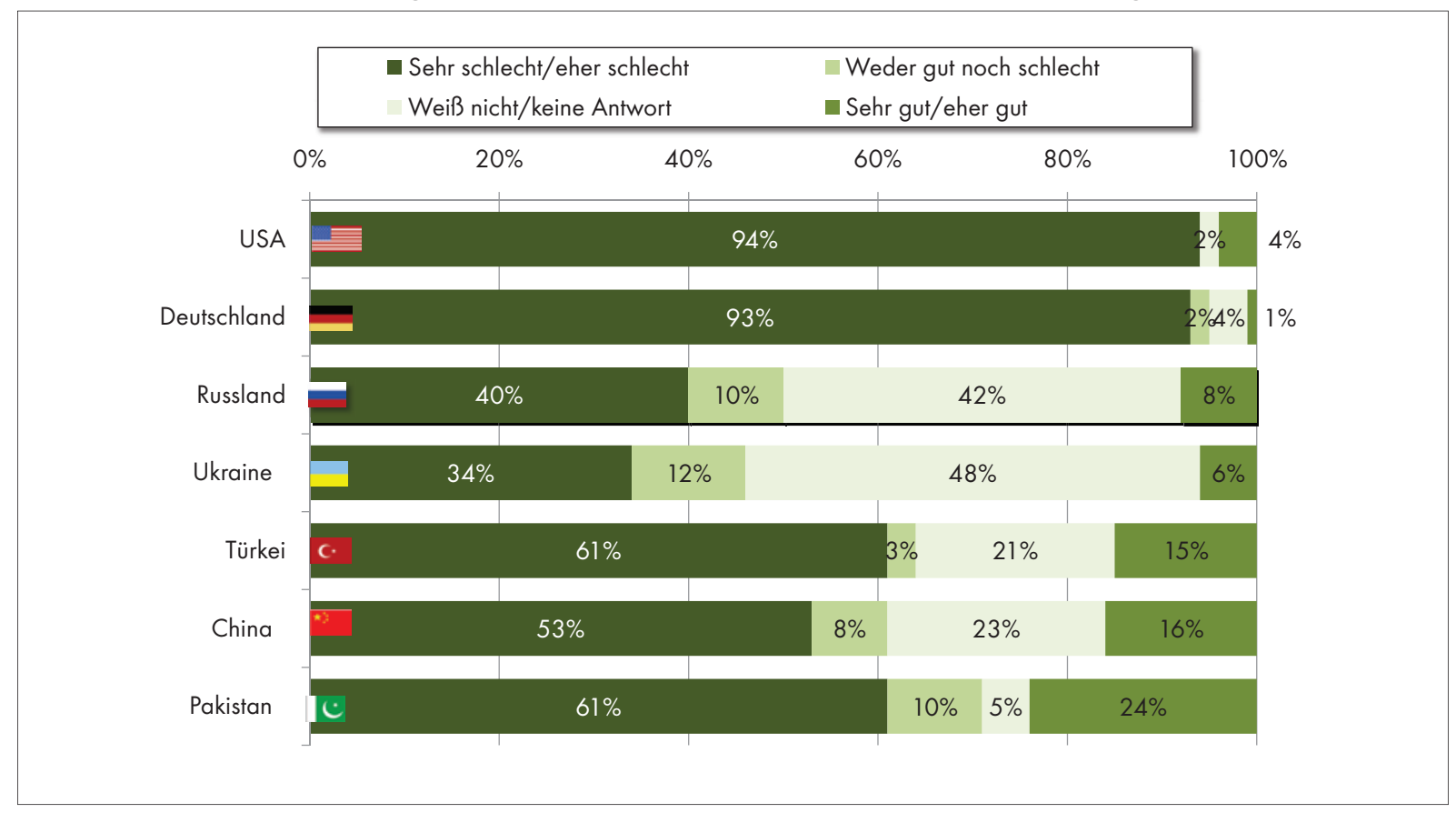

Quelle: Umfragen von WorldPublicOpinion vom Juli 2009,

http://www.worldpublicopinion.org/pipa/pdf/jul09/WPO_Afghan_Jul09_quaire.pdf 
Die Obama-Administration hat beschlossen, die Zahl der US-Truppen in Afghanistan zu erhöhen. Was halten Sie von dieser Entscheidung?

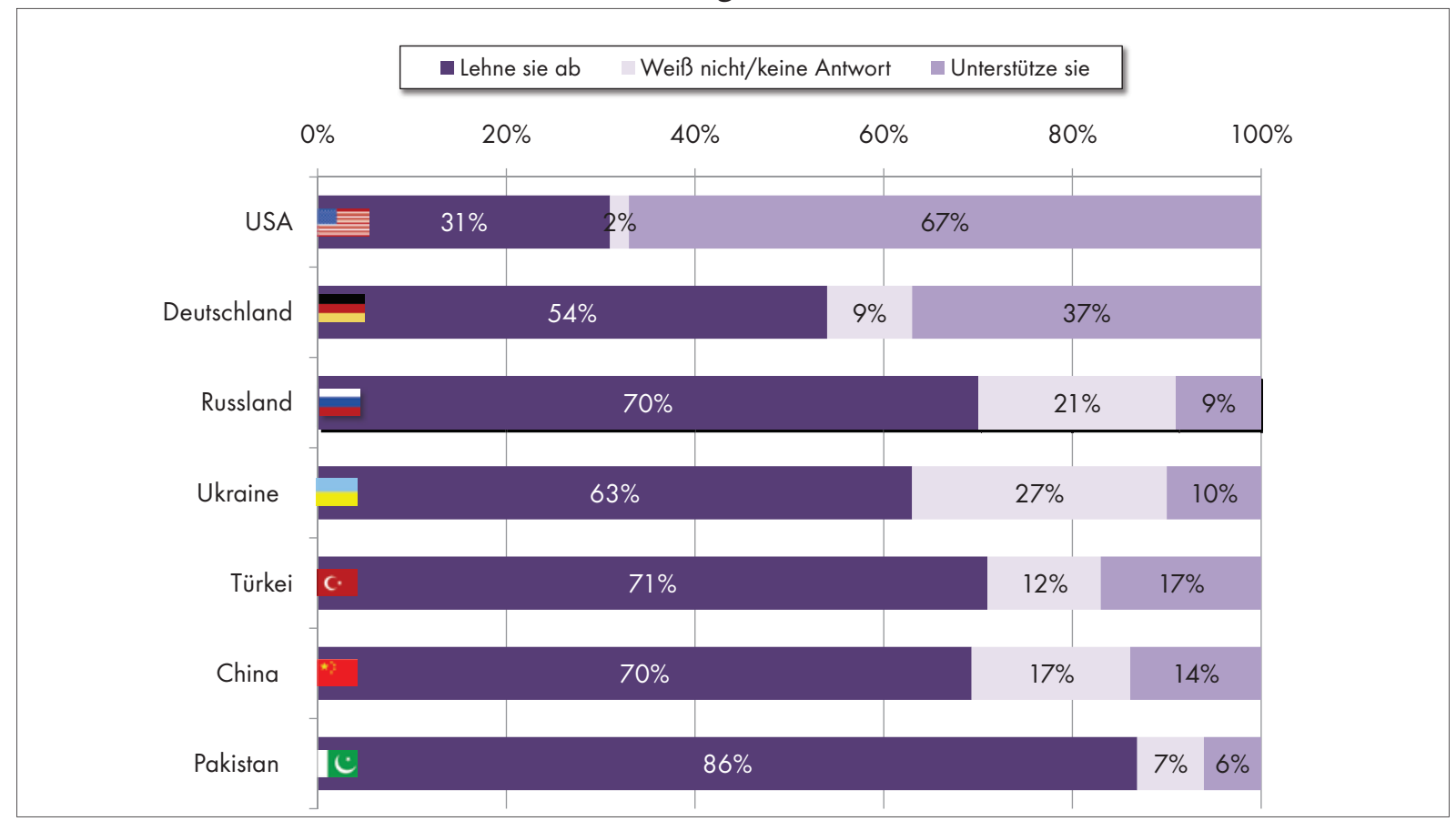

Quelle: Umfragen von WorldPublicOpinion vom Juli 2009,

http://www.worldpublicopinion.org/pipa/pdf/jul09/WPO_Afghan_Jul09_quaire.pdf

Sollten die USA und die NATO Truppen in Afghanistan bis zu einer Stabilisierung der Lage belassen?

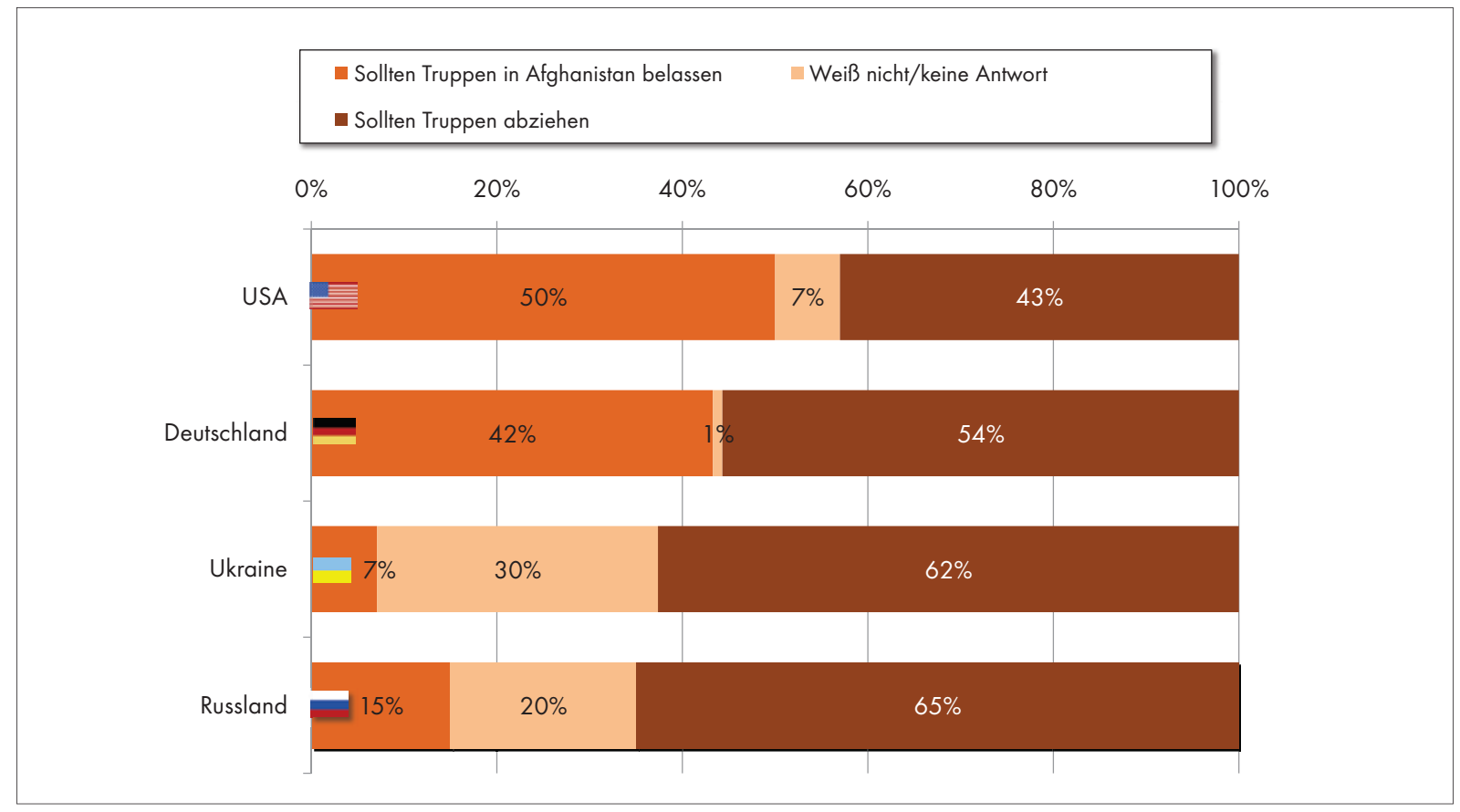

Quelle: Umfragen des Pew Global Attitudes Project, August-September 2009, http://pewresearch.org/assets/pdf/1407.pdf 


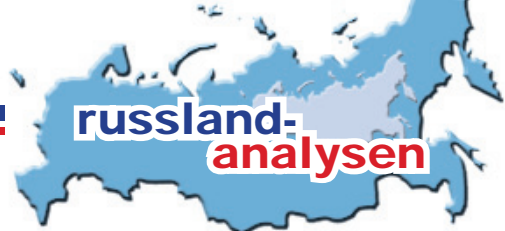

Wäre es eine Bedrohung, wenn islamistische Extremisten die Kontrolle in Pakistan übernehmen würden?

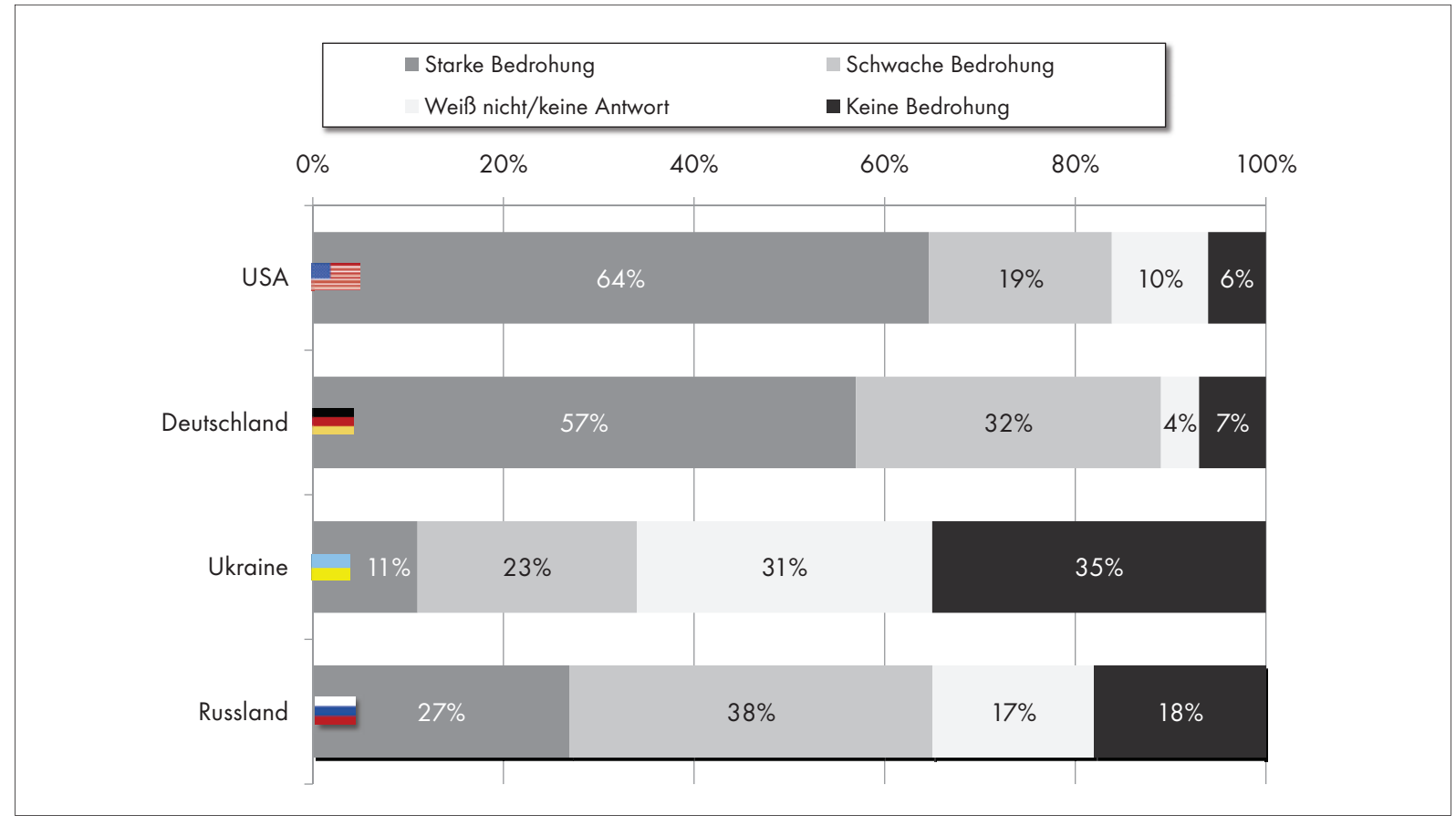

Wäre es eine Bedrohung, wenn die Taliban wieder die Kontrolle in Afghanistan übernehmen würden?

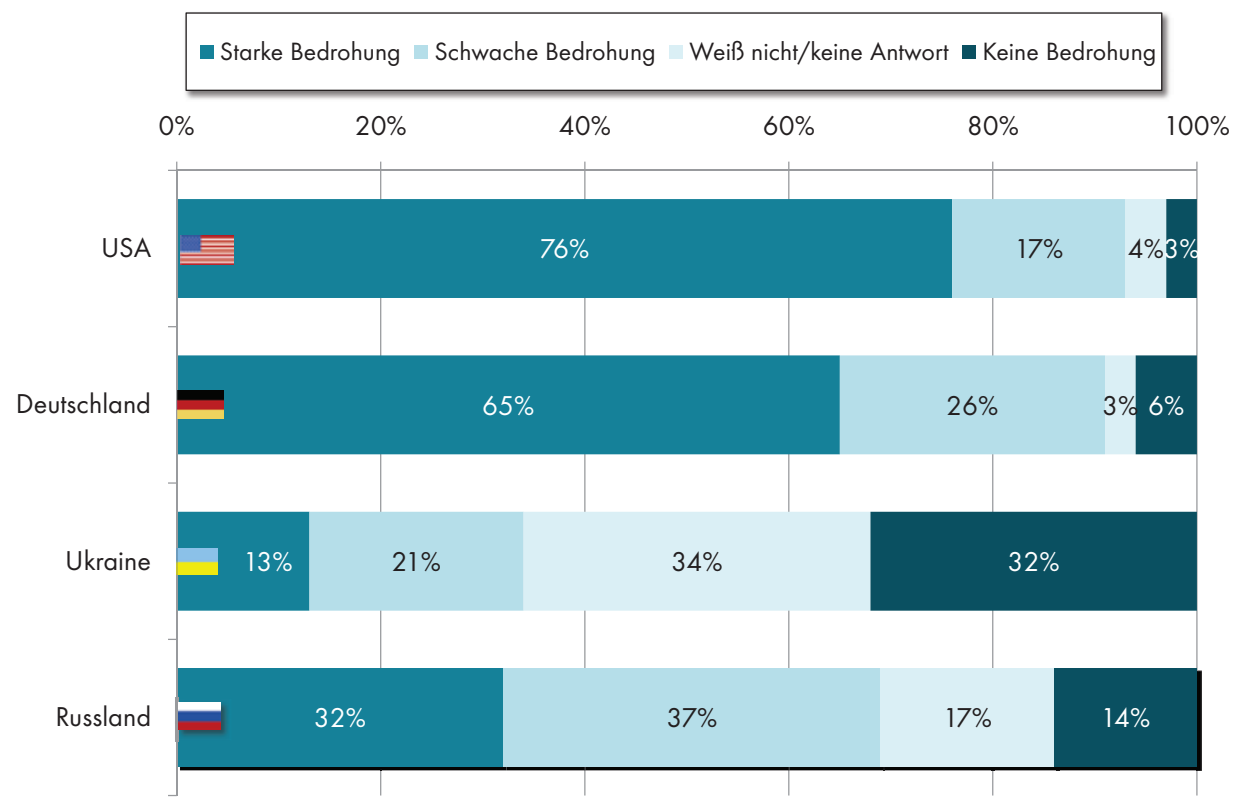

Quelle: Umfragen des Pew Global Attitudes Project, August-September 2009, http://pewresearch.org/assets/pdf/1407.pdf 


\section{Der sowjetische Afghanistankrieg in der russischen Erinnerung}

Welche der folgenden Einschätzungen des Afghanistankrieges (1979-1989) teilen Sie?

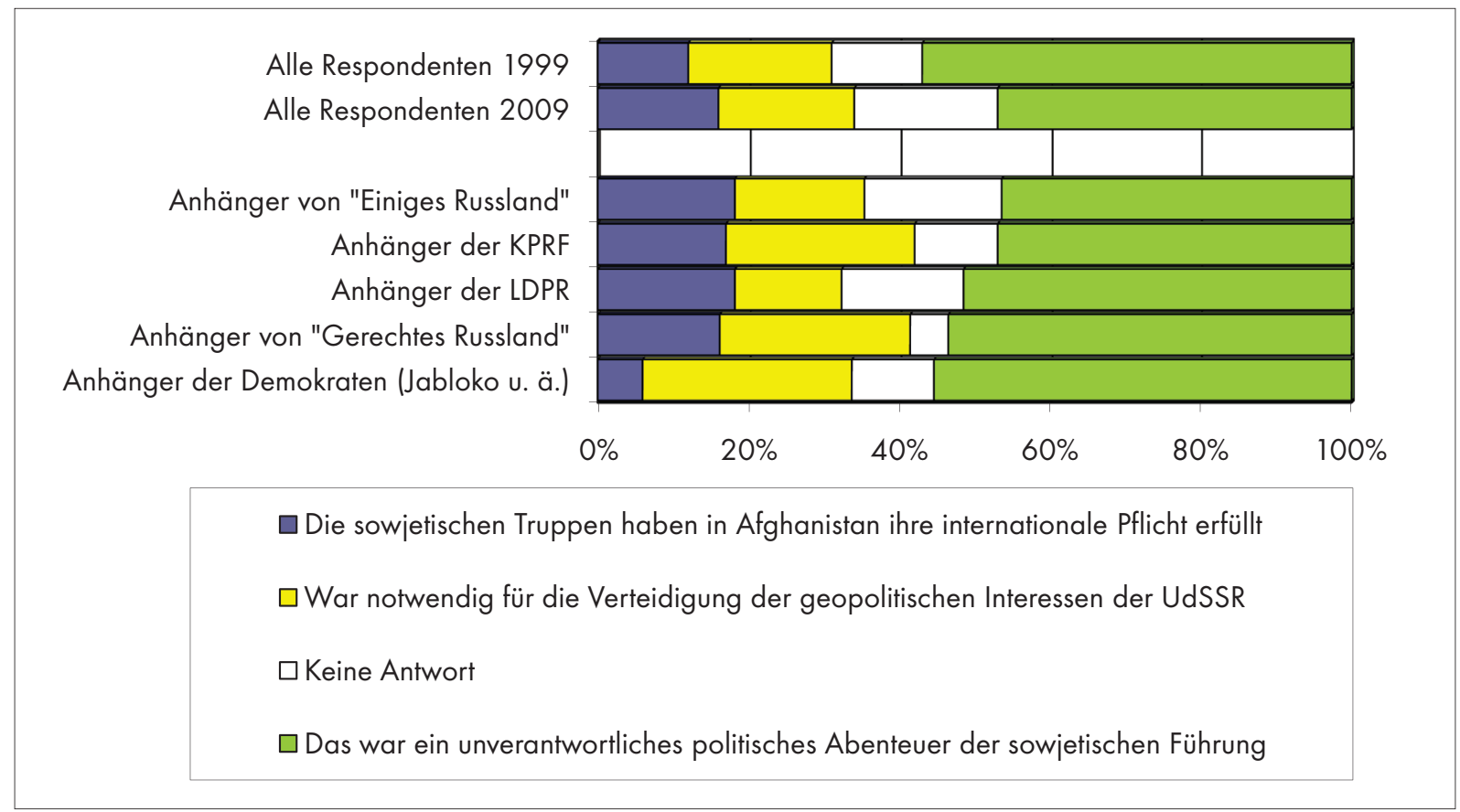

Woher wissen Sie vom Krieg in Afghanistan?

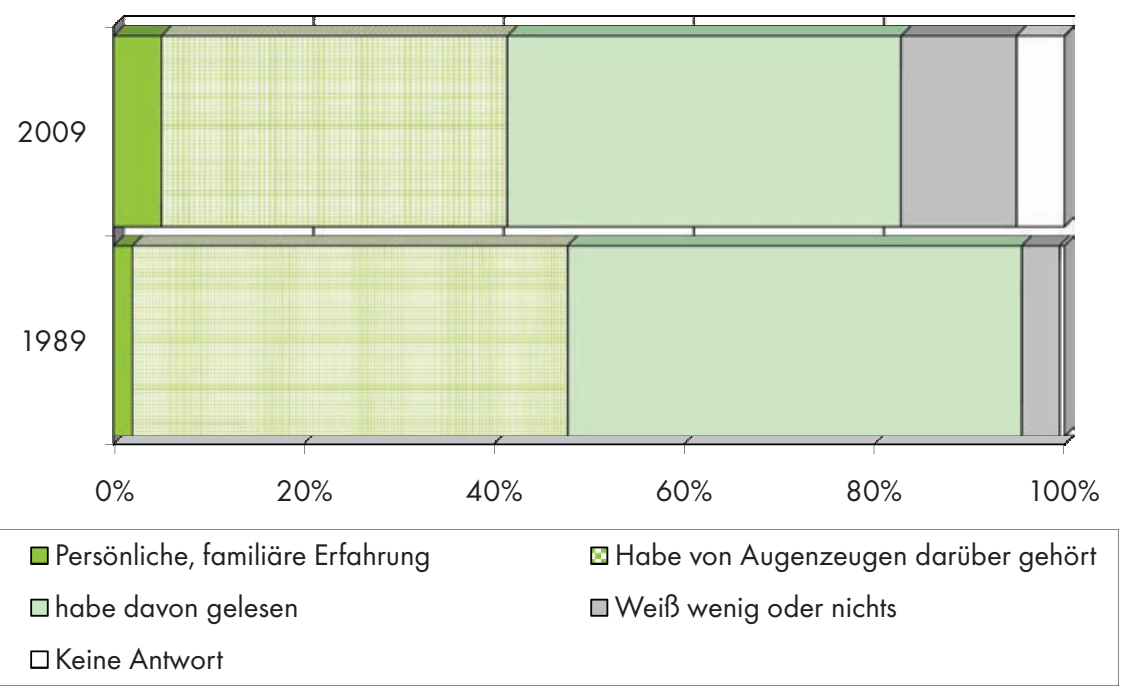

Quelle: Umfragen des WZIOM vom 7.-8. Februar 2009, http://wciom.ru/novosti/press-vypuski/press-vypusk/single/11403.html 


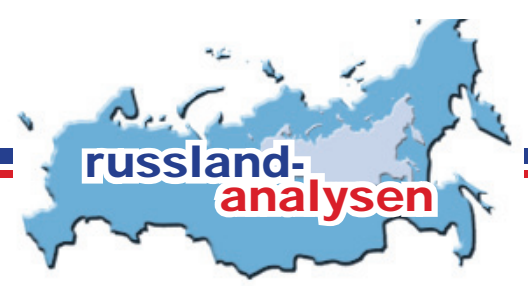

Ost-Ausschuss der Deutschen Wirtschaft

oa

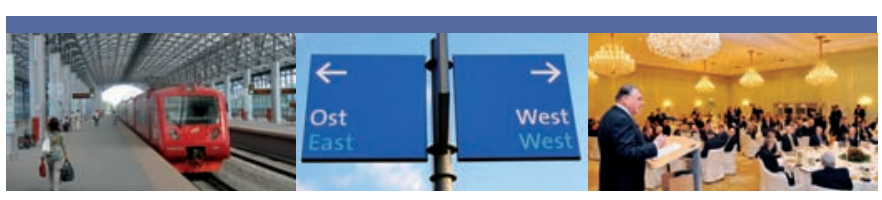

- Der Ost-Ausschuss der Deutschen Wirtschaft arbeitet seit 1952 aktiv daran, die Handelsund Investitionsbedingungen für deutsche Unternehmen in über 20 Ländern des östlichen Europas und Zentralasiens zu verbessern.

Unsere Konferenzen und Fachseminare informieren Sie über aktuelle Entwicklungen zwischen Zagreb und Wladiwostok.
- Wir sind die Stimme der deutschen Wirtschaft in bilateralen Gremien und vernetzen Wirtschaft und Politik durch die Organisation exklusiver Wirtschaftsgespräche und Delegationsreisen.

Mit unseren Projekten fördern wir die marktwirtschaftliche Entwicklung in Osteuropa und investieren in die Aus- und Weiterbildung junger Menschen.

Weitere Informationen zu unseren Veranstaltungen, Publikationen und zur Mitgliedschaft im Ost-Ausschuss finden Sie unter: www.ost-ausschuss.de

\section{Brücke zu den Zukunftsmärkten}

\section{Ost-Ausschuss der Deutschen Wirtschaft}




\section{Lesehinweis}

\section{Kostenlose E-Mail-Dienste der Forschungsstelle Osteuropa auf www.laender-analysen.de}

\section{Caucasus Analytical Digest}

Der Caucasus Analytical Digest bietet einmal monatlich englischsprachige Kurzanalysen sowie illustrierende Daten zu einem aktuellen Thema. Zusätzlich gibt es eine Chronik aktueller Ereignisse.

Abonnement unter: http://www.res.ethz.ch/analysis/cad/

\section{Polen-Analysen}

Die Polen-Analysen bieten zweimal monatlich eine Kurzanalyse zu einem aktuellen Thema aus Politik, Wirtschaft oder Kultur, ergänzt um Grafiken und Tabellen. Zusätzlich gibt es eine Chronik aktueller Ereignisse.

Abonnement unter: http://www.deutsches-polen-institut.de/Newsletter/subscribe.php

\section{Russland-Analysen}

Die Russland-Analysen bieten vierzehntägig eine Kurzanalyse zu einem aktuellen Thema, ergänzt um Grafiken und Tabellen. Zusätzlich gibt es eine Chronik aktueller Ereignisse.

Abonnement unter: fsopr@uni-bremen.de

\section{Russian Analytical Digest}

Der Russian Analytical Digest bietet zweimal monatlich englischsprachige Kurzanalysen sowie illustrierende Daten zu einem aktuellen Thema.

Abonnement unter: http://www.res.ethz.ch/analysis/rad/

\section{Ukraine-Analysen}

Die Ukraine-Analysen bieten zweimal monatlich eine Kurzanalyse zu einem aktuellen Thema aus Politik, Wirtschaft oder Kultur, ergänzt um Grafiken und Tabellen. Zusätzlich gibt es eine Chronik aktueller Ereignisse.

Abonnement unter: fsopr@uni-bremen.de

\section{Zentralasien-Analysen}

Die Zentralasien-Analysen bieten monatlich eine Kurzanalyse zu einem aktuellen Thema aus Politik, Wirtschaft oder Kultur, ergänzt um Grafiken und Tabellen. Zusätzlich gibt es eine Chronik aktueller Ereignisse.

Abonnement unter: zentralasien-analysen@dgo-online.org

\section{Bibliographische Dienste}

Die vierteljährlich erscheinenden Bibliographien informieren über englisch- und deutschsprachige Neuerscheinungen zu Polen, Russland, Tschechischer und Slowakischer Republik, Ukraine sowie zu den zentralasiatischen und kaukasischen Staaten. Erfasst werden jeweils die Themenbereiche Politik, Außenpolitik, Wirtschaft und Soziales.

Abonnement unter: fsopr@uni-bremen.de 